\title{
Biophysical characterization of melanoma cell phenotype markers during metastatic progression
}

\author{
Anna Sobiepanek $^{1}$ (D) $\cdot$ Alessio Paone $^{2}$ (D) $\cdot$ Francesca Cutruzzolà $^{2}$ (D) Tomasz Kobiela $^{1}$ (D)
}

Received: 30 October 2020 / Revised: 30 January 2021 / Accepted: 8 March 2021 / Published online: 17 March 2021

(c) The Author(s) 2021

\begin{abstract}
Melanoma is the most fatal form of skin cancer, with increasing prevalence worldwide. The most common melanoma genetic driver is mutation of the proto-oncogene serine/threonine kinase BRAF; thus, the inhibition of its MAP kinase pathway by specific inhibitors is a commonly applied therapy. However, many patients are resistant, or develop resistance to this type of monotherapy, and therefore combined therapies which target other signaling pathways through various molecular mechanisms are required. A possible strategy may involve targeting cellular energy metabolism, which has been recognized as crucial for cancer development and progression and which connects through glycolysis to cell surface glycan biosynthetic pathways. Protein glycosylation is a hallmark of more than 50\% of the human proteome and it has been recognized that altered glycosylation occurs during the metastatic progression of melanoma cells which, in turn facilitates their migration. This review provides a description of recent advances in the search for factors able to remodel cell metabolism between glycolysis and oxidative phosphorylation, and of changes in specific markers and in the biophysical properties of cells during melanoma development from a nevus to metastasis. This development is accompanied by changes in the expression of surface glycans, with corresponding changes in ligand-receptor affinity, giving rise to structural features and viscoelastic parameters particularly well suited to study by label-free biophysical methods.
\end{abstract}

Keywords Melanoma $\cdot$ Metastasis $\cdot$ Metabolism $\cdot$ Glycosylation $\cdot$ Label-free techniques

\section{Introduction}

The most fatal skin-related cancer worldwide is melanoma, which develops as a result of mutation and uncontrolled proliferation of melanocytes, the skin cells producing the pigment melanin. Most melanoma cells retain the ability to produce melanin, thus they form pigmented (typically brown or black) melanomas. But in rare cases with disrupted melanin synthesis, amelanotic melanoma may occur. The primary site of melanoma development is the skin, but cases of the

Special Issue: COST Action CA15126, MOBIEU: Between atom and cell.

Anna Sobiepanek

asobiepanek@ch.pw.edu.pl

1 Laboratory of Biomolecular Interactions Studies, Chair of Drug and Cosmetics Biotechnology, Faculty of Chemistry, Warsaw University of Technology, Noakowskiego 3, 00-664 Warsaw, Poland

2 Department of Biochemical Sciences "A. Rossi Fanelli”, Sapienza University of Rome, Rome, Italy eye (e.g., uvea), meninges and various mucosal surfaces (e.g., oral mucosa) have also been reported. Many different clinical subtypes of melanoma have been described, among which the most common are: acral lentiginous melanoma (ALM), nodular melanoma (NM), lentigo maligna melanoma (LMM) and superficial spreading melanoma (SSM). Melanoma in early phases (mainly the radial growth phase, RGP) is entirely curable thanks to tissue excision, but if metastasis occurs the chances for patients' survival drastically drops. The spreading of melanoma cells via the lymph nodes and vessels makes it very difficult to eliminate all tumor cells from the organism. Due to rising incidence and mortality rates, it is considered one of the top ten deadliest tumors (Forman et al. 2008; Rastrelli et al. 2014; Dummer et al. 2016; Elder 2016; Dehdashtian et al. 2018; Sobiepanek et al. 2020a).

During the multiple steps of tumor development, cells gain several biological capabilities (the so-called hallmarks of cancer), which distinguish them from normal cells. Basic hallmarks of tumor cells indicate that they are able to produce various growth factors, evade growth suppressors, 
gain replicative immortality, resist apoptosis, avoid immune surveillance, induce angiogenesis, and present invasive growth and formation of metastasis. Moreover, changes in the energy metabolism as well as other signaling pathways are vital parts of cell transformation and tumor formation (Hanahan and Weinberg 2011). Several metabolic pathways have been associated with melanoma metastasis including: (1) alanine, aspartate and glutamate metabolism, (2) glycine, serine and threonine metabolism, (3) arginine and proline metabolism, (4) $\beta$-alanine metabolism, (5) aminoacyl-tRNA biosynthesis, (6) cysteine and methionine metabolism and (7) D-Glutamine and D-glutamate metabolism (Kim et al. 2017). On the other hand, changes in the glycan biosynthesis pathway may lead to the altered glycosylation profile of cells which undergo tumorigenic transformation (Sweeney et al. 2018). The nature of melanoma surface glycans is highly dynamic and changes during differentiation and in response to different stimuli through the action of glycosyltransferases and glycosidases. A crucial role of some specific, modified glycans in various phases of melanoma progression has been documented (Ząbczyńska and Pocheć 2015). These changes comprise an increase in the amount of hypersialilated $\mathrm{N}$-oligosaccharides or presence of short, simple glycans in advanced stages of melanoma (CiołczykWierzbicka et al. 2004). These properties determine glycan interactions with specific ligands, used for their identification and description of the complexes formed (Sobiepanek et al. 2017). A combined targeting of these pathways may help in establishing effective therapies for various tumors. They may also function as a set of diagnostic and prognostic biomarkers.

\section{General differences between melanocytes and melanoma cells}

Melanocytes, which may transform into melanoma cells, originate from the neural crest, not like other skin carcinomas from embryonic epithelial cells. The key feature of the neural crest cells is multipotency and the ability to migrate. This is the main reason for the capability of melanoma cells to cross the basement membrane and metastasize (Donoghue et al. 2008; Sobiepanek et al. 2020b). Contrary to melanocytes, in which melanin synthesis is under strict control (Slominski et al. 2012), the pigment itself plays a specific biological role mainly in the skin protection against harmful factors like solar radiation, reactive species or chemotherapeutics, melanin production is highly dysregulated in melanoma cells (Mishra et al. 2014) and the amount of melanin can be a biomarker of cancer progression (Slominski et al. 2014). Degraded melanin, impaired melanogenesis or the retention of melanin in cells might have a mutagenic influence on the cells with increased melanoma appearance (Śniegocka et al. 2018).

The molecular bases of melanoma progression are the subject of intensive research. Malignant transformation is connected with mutations in genes responsible for cell proliferation and apoptosis, epigenetic changes, loss of adhesion ability or production of autocrine growth factors, which disturb the signal transduction pathways in melanocytes. For example, the first melanoma oncogene described in 1984 was the NRAS protein, which activates the mitogenactivated protein kinases MAPK) cascade (Pokrywka and Lityńska 2012). Currently, mutations in four different proteins (BRAF, GNAQ/GNA11, cKIT, NRAS) are well known to occur during melanoma formation, and affect the MAPK pathways by disrupting the healthy cells' response to the emerging oncogenes. Point mutations in the v-Raf murine sarcoma viral oncogene homolog (BRAF) are present in $35-50 \%$ of melanoma cases, while in Neuroblastoma RAS viral oncogene homolog (NRAS) only in 10-25\% cases, and there are loss of function mutations affecting Neurofibromin 1 (NF1) in approximately $15 \%$ of melanoma cases. Although these changes are considered as tumor markers, the unequivocal molecular marker of melanoma has not been defined yet (Scolyer et al. 2011; Fischer et al. 2018).

Metabolic changes observed in melanoma include mainly differences in the signaling pathways responsible for the cell growth, mobility, proliferation and/or the cell survival (e.g., RAS/RAF/MAPK, PI3K/AKT/mTOR and p53/Bcl-2 pathways) as compared to the normal melanocytes. On the other hand, the formation of the tumor foci in distant tissues from the primary tumor occurs through the mechanism of epithelial-mesenchymal transition (EMT). This allows the polarized epithelial type cells to modify their phenotype to mesenchymal. The cells' adhesion ability declines, the apical-basal polarity is lost and at the same time cells gain increased mobility. The EMT process in addition to the increased mobility of cells is also accompanied by disorders in the control of the cell cycle, differentiation processes, the development of resistance to apoptosis, as well as the increased motility of cells. Angiogenesis provides a constant supply of nutrients to tumor cells, but also provides a channel through which transformed mesenchymal cells detach from the mass of the tumor and move to distant organs. Mesenchymal cells after colonizing new tissue return to the epithelial phenotype on the basis of the mesenchymal-epithelial transition (MET) mechanism (Kalluri and Weinberg 2009; Nakamura and Tokura 2011; Fenouille et al. 2012; Heerboth et al. 2015).

The significance of metabolic reprogramming in cancer initiation, maintenance, as well as progression is important. Especially when taking into consideration that this tumor is metabolically heterogeneous and capable of adapting to multiple conditions as well as to the use of a variety of fuels. 
There is also growing evidence that the metabolic phenotypes of melanoma cells strongly depend on not only the intrinsic oncogenic pathways but also on extrinsic factors present in the tumor microenvironment, a complex matrix composed of various cells (Hanahan and Weinberg 2011; Fischer et al. 2018).

\section{Metabolic pathways in melanoma}

It is obvious that the central carbon metabolism is the most important process occurring in all types of cells. It consists of glycolysis, the pentose phosphate pathway (PPP) and tricarboxylic acid (TCA) cycle. Depending on the cell type (normal differentiated cells or cancer cells) glucose metabolism varies significantly. Normal cells under normoxic conditions uptake glucose, which then undergoes the process of glycolysis in the cytosol to produce pyruvate and adenosine triphosphate (ATP) molecules. Ten intracellular enzymatic reactions are required to degrade glucose into pyruvate, then the decarboxylation of pyruvate to acetyl coenzyme A (acetyl-CoA) by mitochondrial pyruvate dehydrogenase (PDH) occurs and acetyl-CoA enters the tricarboxylic acid cycle (TCA cycle, also called Krebs cycle) in mitochondria (Fischer et al. 2018). The TCA cycle produces nicotinamide adenine dinucleotide (NADH) and reduced flavin adenine dinucleotide $\left(\mathrm{FADH}_{2}\right)$, which fuels oxidative phosphorylation (OXPHOS) by the electron transport chain (ETC) found in the mitochondrial inner wall to form ATP (Courtnay et al. 2015). Five complexes are responsible for the OXPHOS in mitochondria: ubiquinone oxidoreductase (complex I), succinate dehydrogenase (complex II), ubiquinol-cytochrome c oxidoreductase (complex III, or cytochrome bc1 complex), cytochrome c oxidase (complex IV) and ATP synthase (complex V) (Sharma et al. 2009). On the other hand, under hypoxic conditions cells rely more on glycolysis and lactic fermentation to fulfill their energy needs. This requires the transformation of glucose to pyruvate, which is then reduced to lactate by the enzyme lactate dehydrogenase (LDH), using the reducing equivalents carried by NADH (Fischer et al. 2018). OXPHOS is significantly more efficient at generating ATP than glycolysis. The total net gain of energy from a single molecule of glucose is 2 molecules of ATP gained via glycolysis or 36 ATP molecules by means of glycolysis/ OXPHOS (Courtnay et al. 2015).

Nevertheless, tumor cells (including melanoma) preferentially metabolize at high rates glucose into lactate regardless of the oxygen availability, but at the same time, they are able to gather sufficient materials required for cell proliferation. This phenomenon is known as aerobic glycolysis or the "Warburg effect" (Fischer et al. 2018). Under normoxic conditions, about $25 \%$ of pyruvate enters the mitochondria of melanoma cells, whereas during hypoxic conditions this value is limited to only 7\% (Scott et al. 2011). However, the mitochondria of melanoma cells stay functional and may additionally support the growth and progression of the tumor (Ruocco et al. 2019; Avagliano et al. 2020). Melanoma cells with KRAS or BRAF mutations have increased glucose uptake as well as higher glycolysis, but they are also able to survive in low glucose conditions in comparison with normal cells (Haq et al. 2013; Courtnay et al. 2015). The cell-surface GLUT-1, a member of the GLUT family of the intracellular glucose uptake membrane transport proteins, is upregulated during the reprogrammed metabolism of many tumors including melanoma and can be associated with a high tumor grade (Ruocco et al. 2019). Apart from the increased glucose consumption, metabolic rewiring in melanoma enhances the reliance on glutamine utilization (Ratnikov et al. 2017).

The activation of intrinsic signaling pathways (like MAPK or PI3K/Akt/mTOR) drives the utilization of the Warburg phenotype in melanoma cells. The activation of these signaling pathways increases the expression of the hypoxia-inducible factor $1 \alpha(H I F 1 \alpha)$, v-MYC avian myelocytomatosis viral oncogene homolog $(M Y C)$ and microphthalmia-associated transcription factor (MITF) (Kumar et al. 2007; Parmenter et al. 2014; Ratnikov et al. 2017). HIF1 $\alpha$ is the central regulator of glycolysis, cancer metabolism and cancer cell proliferation. It has a significant influence on several gene products associated with metabolism, such as: glucose transporter 1 (GLUT1), glucose transporter 3 (GLUT3), glyceraldehyde-3-P-dehydrogenase (GAPDH), hexokinase $1(H K 1)$, hexokinase 2 (HK2), lactic dehydrogenase A ( $L D H A)$, pyruvate kinase $\mathrm{M}(P K M)$, pyruvate dehydrogenase $(P D H)$, pyruvate dehydrogenase kinase 1 $(P D C 1)$, carbonic anhydrase $(C A)$, monocarboxylate transporter 4 (MCT4). For example, HIF1 $\alpha$ activates PDK, which inhibits PDH and in this way prevents the pyruvate from entering the TCA cycle for use in OXPHOS, thus increasing lactate production (Kim et al. 2006; Stubbs and Griffiths 2010; Courtnay et al. 2015). In particular, the glycolysis induced by HIF $1 \alpha$ is strictly linked to GLUT- 1 overproduction and its localization in the membrane. The higher level of the protein GLUT-1 in cutaneous melanoma in comparison with melanocytic nevi, is positively correlated to mitotic activity, melanoma progression and metastasis (Ruocco et al. 2019). Glucose uptake may also be stimulated by MYC, which transcriptionally activates LDH, GLUT-1 and HK2 (Avagliano et al. 2020).

In various tumors, a significant amount of the glycolytic carbon is redirected into the synthesis of the nonessential amino acid serine. The Serine Synthesis Pathway (SSP) includes various enzymes beginning with phosphoglycerate dehydrogenase (PHGDH), followed by phosphoserine aminotransferase 1 (PSAT1) and phosphoserine phosphatase (PSPH). Serine, both imported from the tumor microenvironment (TME), as well as 
endogenously generated by the SSP, is then fueled into the one-carbon metabolism (OCM), through the activity of the key enzyme-serine hydroxymethyltransferase (SHMT). SHMT plays a relevant role in the metabolic reprogramming of cancer cells due to its overexpression. Two SHMT genes were identified in humans: SHMTI encoding the cytoplasmic isozyme (SHMT1) and SHMT2 encoding the mitochondrial isozyme (SHMT2). Serine anabolism through OCM fuels the de novo biosynthesis of purines and pyrimidines as well as the production of antioxidant molecules like glutathione (GSH) (Amelio et al. 2014; Paone et al. 2014; Marani et al. 2016; Paiardini et al. 2016).

The control of oxidative stress plays a pivotal role in the growth of cancer cells. The Warburg phenotype is predominantly driven by a transcriptional co-factor that regulates multiple mitochondrial genes, peroxisome proliferator-activated receptor $\gamma$ 1- $\alpha$ (PPARGC1A, also known as PGC-1 $\alpha$ ). Melanoma cells presenting a higher expression of PGC- $1 \alpha$ are able to tolerate oxidative stress to a significantly greater extent than cells with a low expression of this factor (Vazquez et al. 2013). That is because PGC-1 $\alpha$ can regulate reactive oxygen species (ROS) levels by inducing the expression of several enzymes involved in their detoxification, mainly superoxide dismutase 2 (SOD2) and glutathione peroxidase 1 (GPX1) (Austin and St-Pierre 2012; Luo et al. 2016). The decrease sensitivity to ROS, produced mainly during OXPHOS, is beneficial for melanoma cells, due to the fact that tumor cells during metastasis experience intense oxidative stress (Fischer et al. 2018; Avagliano et al. 2020). The nuclear peroxisome proliferator-activated receptors (PPARs) participate in the regulation of cellular metabolism, inflammation and in melanogenesis. They are a focal regulatory point at the intersection of AMP-dependent protein kinase (AMPK), mammalian target of rapamycin (mTOR) and PGC-1 $\alpha$ signaling pathways (Grabacka et al. 2020). PPAR $\gamma$ activates the transcription of multiple genes involved in lipid synthesis and accumulation, and likewise it influences glucose uptake. PPAR $\alpha$ is responsible for the transactivation genes of the peroxisomal and mitochondrial fatty acid oxidation as well as ketogenesis. The cooperation between PPAR $\gamma$ and $\alpha$-melanocyte-stimulating hormone $(\alpha-\mathrm{MSH})$ signaling results in enhanced melanogenesis in both melanocytes and melanoma cells (Grabacka et al. 2017). PPARs are also important transcription factors taking part in the regulation of ketogenesis, which requires efficient mitochondrial $\beta$-oxidation of fatty acids. The formation of ketone bodies [including $\beta$-hydroxybutyrate (bHB), acetoacetate and acetone] supply additional substrates for the energy metabolism for certain cells from peripheral tissues apart from glucose and glutamine metabolism. Typically, melanoma cells utilize large amounts of glucose, however, it is under intensive investigation whether this monosaccharide could be replaced by ketone bodies (Grabacka et al. 2016a, b).

Differences in cell metabolism are also associated with melanogenesis. This pathway requires three melanocyticspecific enzymes: a melanosomal membrane-bound glycoprotein called tyrosinase and the tyrosinase-related proteins (TRP1 and TRP2); which are involved in the conversion of tyrosine to melanin (Ortonne and Ballotti 2000; García-Borrón and Solano 2002). The oxidoreduction reactions also produce several intermediates including toxic compounds like quinones, semiquinones and ROS that influence the behavior of normal and malignant melanocytes and their surrounding microenvironment (Slominski et al. 2014). Melanogenesis is mediated by several melanogenic signaling pathways like p38 MAPK signaling, the cyclic adenosine monophosphate- (cAMP-) mediated pathway, the protein kinase C- (PKC-) mediated pathway, PI3K/AKT signaling, and the p44/42 MAPK pathway. That is why while affecting melanization it can directly interfere with pro-proliferation and antiapoptotic regulation; thus, melanogenesis is directly connected with the prognosis of melanoma development (Hwang et al. 2019). Furthermore, it has been found that the induction of melanogenesis in melanoma cells is associated with a dramatic increase in nuclear HIF1 $\alpha$ expression accompanied by the upregulation of HIF $1 \alpha$-dependent genes involved in the regulation of glucose metabolism, angiogenesis and stress responses (Slominski et al. 2014).

Signals produced by the TME can also influence melanoma plasticity through changes in the epigenetic state to force dynamic differentiation and de-differentiation. The microenvironment-mediated epigenetic regulation of gene expression includes stress signals like $\mathrm{pH}$, hypoxia, radiation, stiffness, as well as interfacial stress, which constrain a conversion of melanoma cell to a stem cell-like phenotype (Lee et al. 2020). The hypothesis of the occurrence of MTSC (melanoma tumor stem cells) presenting the CD 271 (neurotrophin receptor) antigen, responsible for metastasis occurring, has been thoroughly verified (Boiko et al. 2010). On the other hand, an oncogenic transformation may force the adaptation of cancer cells to the conditions occurring in the TME. This is done by a metabolic phenotype switch that can significantly influence the tumor microenvironment. Due to the complex TME structure, inefficient nutrient supply and oxygen delivery inside of the tumor takes place as well as poor waste removal from the tumor (Ratnikov et al. 2017; Avagliano et al. 2020; Bader et al. 2020). The growth and progression of solid tumors, like cutaneous melanoma, as well as therapeutic resistance are strongly dependent on the metabolic crosstalk between cancer cells and the TME (Ruocco et al. 2019). Lactate secretion not only drastically alters the TME, but also facilitates angiogenesis, promotes metastasis and suppresses the immune system (RomeroGarcia et al. 2016). For many years, the production of lactic 
acid was believed to acidify the cytoplasm of cells, affecting the tumor intracellular $\mathrm{pH}(\mathrm{pHi})$ as well as extracellular $\mathrm{pH}(\mathrm{pHe})$. In case of tumor extracellular $\mathrm{pH}$, this was confirmed by the discovery of enzymes located on the cell surface membrane, which catalyze the reversible interconversion of $\mathrm{CO}_{2}$ to $\mathrm{HCO}_{3}{ }^{-}$and $\mathrm{H}^{+}$, e.g., the carbonic anhydrase IX (CAIX). Carbonic anhydrases are cancer-associated cell surface zinc metalloprotein enzymes (a family of 16 isoforms), which play the key role in maintaining low tumor pHe. The pHe in healthy tissues is between 7.35 and 7.45 and it is tightly regulated to sustain the normal physiology and cellular metabolism. In case of tumor cells, $\mathrm{pHe}$ is acidic (6.3-7.0), reflecting the dysregulation of the acid-base homeostatic mechanisms present in solid tumors. However, it turned out that the intracellular pHi is usually neutral or alkaline in both normal and cancerous cells, which is why only $\mathrm{pHe}$ and its controlling factors are assumed to be potential therapeutical targets in oncology. CAIX consists of an extracellularly facing catalytic domain, an $\mathrm{N}$-terminal proteoglycan-like (PG) domain, a single transmembrane domain and a short intracellular C-terminal tail, where the $\mathrm{N}$-terminal PG domain of CAIX is unique within the CA family and is important in the assembly of focal adhesion contacts during cell migration (Lee and Griffiths 2020).

In addition, adherent cell types express various glycosylated cell receptors responsible for focal adhesion. Disturbance in glucose availability affects glycosylation, which is a hallmark of more than $50 \%$ of the human proteome responsible for the development, growth and survival of cells and organisms (Christiansen et al. 2014; Pinho and Reis 2015). Many studies reveal that cancer development and progression including EMT and invasion is accompanied by changes in the glycosylation patterns of cell surface and secreted glycoproteins (Munkley and Elliott 2016). Moreover, a growing amount of data has shown that different levels of oxygen tension lead to a markedly altered glycosylation, resulting in altered glycan-receptor interactions (Silva-filho et al. 2017).

\section{Glycosylation basics and melanoma}

Glycosylation is the most common post-translational modification in cells, present in more than half of proteins synthesized (forming glycoproteins and proteoglycans) and a subset of lipids (glycosphingolipids and glycosylphosphatidylinositol anchors) (Vasconcelos-dos-Santos et al. 2015). To successfully generate these carbohydrate-associated modifications a coordinated effort of a complex array of enzymes, organelles and other factors is needed, or else the genetic and non-genetic alterations may facilitate the development of neoplastic transformation (Stowell et al. 2015). For over 35 years, the abnormal glycosylation of peptides, proteins and membrane lipids has been observed in various diseases such as diabetes, rheumatoid arthritis, multiple sclerosis and also all types of human cancers. Despite comprehensive research into the role of cancer glycobiology, including the structure and function of carbohydrates (Ghazarian et al. 2011; Dall'Olio 2012; Nardy et al. 2016), the specific characteristics of metastatic cell glycans of different types of tumors remain the subject of many studies (Häuselmann and Borsig 2014; Taniguchi and Kizuka 2015). Particular attention in such glycosylation studies is focused not only on the rapid growth and high malignancy of tumors including melanoma, but also on the identification of biomarkers or design of anti-cancer drugs.

Proteins undergo glycosylation as either a co-translational or post-translational modification (Christiansen et al. 2014). The glycosylation process involves attaching sugar groups in the form of monosaccharides or oligosaccharides to specific amino acids of the polypeptide chain (Stowell et al. 2015). So far, about 250 enzymes have been discovered that enable the glycosylation process; due to their functions, they can be divided into two groups: glycosyltransferases and glycosidases. The first group is responsible for the attachment of new sugar residues, while the second group hydrolyzes glycosidic bonds and detaches monosaccharides to trim and refine glycan side chains (Ząbczyńska and Pocheć 2015).

The majority of glycans are comprised of ten monosaccharide building blocks, which are glucose (Glc), galactose (Gal), fucose (Fuc), mannose (Man), xylose (Xyl), $\mathrm{N}$-acetylglucosamine (GlcNAc), $\mathrm{N}$-acetylgalactosamine (GalNAc), glucuronic acid (GlcA), iduronic acid (IdoA) and 5- $\mathrm{N}$-acetylneuraminic acid (Neu5Ac or sialic acid) (Stowell et al. 2015). The five classes of glycans which can be created are: $\mathrm{N}$-glycans, $O$-glycans, glycosaminoglycans (GAGs), glycosphingolipids (GSLs), and glycosylphosphatidylinositol (GPI) anchor (Marsico et al. 2018). The carbohydrate chain can be covalently bound to the protein through an oxygen atom in case of the O-glycosylation [the GalNAc residue is attached to the hydroxyl group of serine (Ser) or threonine (Thr)] or via a nitrogen atom for the $\mathrm{N}$-glycosylation (the GlcNAc residue is attached to asparagine (Asn) in the amino acid sequence Asn-X-Ser/Thr, where $\mathrm{X}$ can be any amino acid except proline) (Christiansen et al. 2014). In case of glycosphingolipids, they are initiated by glucose addition to ceramide on the outer membrane of the ER and Golgi apparatus, and then the glycan is flipped inside these organelles to be extended. In general, the thick layer of all kinds of glycoconjugates present on the cell surface form the glycocalyx, however, some glycans secreted to the extracellular medium can also be incorporated into the extracellular matrix (ECM) (Vasconcelos-dos-Santos et al. 2015).

The donor substrates for the glycosylation process are derived in some part from the intracellular degradation of glycoconjugates in lysosomes, but mainly from extracellular glucose. During the glycolysis process, a side path may be activated, in which the glucose metabolite can be diverted 
to the Hexosamine Biosynthetic Pathway (HBP). Normal cells direct $2-5 \%$ of glucose to the HBP pathway, while for tumor cells this amount is altered mainly by the fact that cancer cells utilize far more glucose than normal cells (Akella et al. 2019). Fructose-6-phosphate (Fru-6P) can be converted to glucosamine-6-phosphate (GlcN-6P) by glutamine fructose-6-phosphate aminotransferase (GFAT) using glutamine (Gln) as an amine donor. Next, GlcN-6P is further metabolized to uridine-5'-diphosphate- $N$-acetylglucosamine (UDPGlcNAc), which serves as a major substrate for several kinds of glycosylations (Vasconcelos-dos-Santos et al. 2015). The glycan biosynthesis carries on with the addition of UDPGlcNAc or its derivatives to dolichol phosphate (in case of $\mathrm{N}$-/O-glycans) or ceramide (Cer; in case of glycosphingolipids) (Stanley et al. 2017; Schnaar and Kinoshita 2017). The process of $N$ - and $O$-glycan oligosaccharide synthesis takes place on the membrane of the endoplasmic reticulum (ER). After several enzyme activities, the glycan structure bonds to the folded protein and is directed to the Golgi apparatus. There, the $\mathrm{N}$-glycan takes its final form of a pentasaccharide core with high mannose chains, and hybrid or complex type side chains. In case of the $O$-glycans, they are built from different types of cores ( $O$-GalNAc, $O$-Man, o-Fuc and o-Glc) with a few side chains (Vasconcelos-dos-Santos et al. 2015).

The altered glycosylation of cell-surface proteins, which is observed at all stages of cancer progression, results in the increased growth, proliferation and survival of cells as well as gives cancer cells the ability to migrate and invade. The accumulation of genetic and epigenetic changes results in dysregulated transcription, which causes an altered expression and also altered activity or the mislocalization of enzymes engaged in the glycan biosynthesis pathway. Typical cancer-associated changes in glycosylation include: (1) the overexpression and underexpression of naturally occurring glycans, (2) the expression of glycans normally restricted to embryonic tissues, (3) the appearance of incomplete or truncated structures and also (4) the appearance of novel structures (less frequently) (Link-Lenczowski and Lityńska 2011; Hoja-Łukowicz et al. 2017). Although it is well known that aberrant glycans are present on various cancer cells, the regulation of glycosylation patterns in different cancers is still largely unknown (Sweeney et al. 2018). In case of melanoma, the most common modification is a higher expression of $\beta 1,6$-branched $\mathrm{N}$-linked glycans (responsible for a significant enhancement of cell motility through the ECM substrates) caused by the increased transcription and activity of $\beta 1,6-\mathrm{N}$-acetylglucosaminyltransferase $\mathrm{V}$ (GnT-V). Another upregulated enzyme responsible for the increased expression of bisected $N$-glycans is $\beta 1,4-N$ acetylglucosaminyltransferase III (GnT-III), which may promote cancer progression. Likewise, the enhanced activity of sialyltransferases such as $\alpha 2-3$ and $\alpha 2-6$ was observed in melanoma cells. Accelerated cell-surface sialylation and/ or differences in the position of sialic residues, including $\alpha 2,8$-linked polysialic acid (PolySia), are responsible for the modulation of cell recognition, cell adhesion and cell-cell signaling. The accumulation of the negative charge in the form of sialic acid content on the surface of the cell membrane causes repulsion between cells, reduces tumor cell anchorage to ECM components, and finally allows tumor cells to migrate from the tumor site. The truncation of serine/threonine $O$-linked glycans with T (Gal $\beta 1-3 \mathrm{GalNAc}-$ $\alpha 1-O$-Ser/Thr), Tn (GalNAc- $\alpha 1-O-S e r / T h r)$, and sialyl-Tn (Sia $\alpha 2-6 \mathrm{GalNAc}-\alpha 1-O-\mathrm{Ser} / \mathrm{Thr}$; sTn) mucin antigens was only observed in tumor tissues. Thus, it may be a good diagnostic marker, but not a prognostic marker due to the presence of $O$-glycan changes occurring independently from the cancer progression stage (Lityńska et al 2001; CiołczykWierzbicka et al. 2004; Przybyło et al. 2007; Pinho and Reis 2015; Lucena et al. 2016; Hoja-Łukowicz et al. 2017).

Melanoma cells also show an increase in the expression of the cell-adhesion molecules (CAMs), which are receptors located on the cell surface and involved in the binding of cell-to-cell and cell-to-ECM. The major difference in their expression between normal and tumor cells was observed in case of proteins like L1 cell-adhesion molecule (L1-CAM), melanoma CAM (MCAM), activated leucocyte CAM (ALCAM), vascular CAM-1 (VCAM-1), intercellular adhesion molecule 1 (ICAM-1), and integrins as well as cadherins. Integrins are highly glycosylated proteins responsible for ligand binding to for example fibronectin and collagen, complexation with other membrane proteins and signal transduction by recruiting the focal adhesion kinase (FAK) or Src family kinases. Thus, integrins can initiate pro-survival but also pro-apoptotic signals. They play an important role at every stage of cancer development, where disturbance in their adhesion is caused by aberrant N-glycosylation. Compared to normal melanocytes, melanoma cells show overexpression of $\alpha 1 \beta 1, \alpha 2 \beta 1, \alpha 3 \beta 1, \alpha 4 \beta 1, \alpha 5 \beta 1$, $\alpha 6 \beta 4, \alpha v \beta 3 \alpha v \beta 6$ and a loss of $\alpha 6 \beta 1$ integrins. It is worth emphasizing that some of these changes are characteristics of the metastasis process, e.g., integrin $\alpha v \beta 3$ is considered a marker of the RGP-to-VGP transition. In contrast, $\alpha 1 \beta 1$ and $\alpha 2 \beta 1$ integrins occur only on the surface of metastatic melanoma cells but $\alpha 3 \beta 1$ is present on both types of melanoma cells: primary and metastatic (Link-Lenczowski and Lityńska 2011; Läubli and Borsig 2019). The cadherins are a different type of cell surface protein and play a significant role in epithelial intercellular adhesion. Disturbances in cadherin adhesion may lead to the formation of a tumor originating from epithelial cells: normal melanocytes express E-cadherin and P-cadherin, where E-cadherin ensures the adhesion of melanocytes to keratinocytes (Przybyło et al. 2002; Ciołczyk-Wierzbicka et al. 2004). This contact enables melanin transfer from melanocytes to keratinocytes through three different mechanisms (Ando et al. 2012), one 
of which involves the formation of a structure called a pigmentation synapse (the melanocyte-keratinocyte adhesion site), where the engagement of surface glycans and lectins is a factor responsible for melanin transfer (Van Den Bossche et al. 2006). During oncogenesis, keratinocyte control over melanocytes is reduced and $\mathrm{N}$-cadherin seems to replace the normal E-cadherin especially in tumor cells with a high level of invasiveness. This process is known as the cadherinswitch. The increasing amount of $\mathrm{N}$-cadherin with a simultaneous loss of the ability to produce E-cadherin is a factor that causes a break in the binding between melanocytes and keratinocytes. The effect of this phenomenon is the acquisition of new adhesion properties by these cells. N-cadherin significantly affects the strength of intercellular and intracellular interactions. However, N-cadherin not only initiates the binding between cancer cells of melanocytic origin and fibroblasts or epithelial cells, but also supports the survival and migration of cancer cells. The switch in cadherins is considered to be the most likely cause of tumor formation (Przybyło et al. 2002; Ciołczyk-Wierzbicka et al. 2004; Sobiepanek et al. 2020a). This phenomenon could also be associated with the production of glycosylation structures known as $\beta 1,6$-branched oligosaccharides. These structures are not produced by normal melanocytes or by cells of early melanoma in situ, but characterize invasive and metastatic tumors. The aberrant expression of $\beta 1,6$-branched oligosaccharides is associated with the increased melanin production, autophagy and high invasive potential of melanoma cells, while in normal melanocytes the accumulation of melanin would cause apoptosis (Lazova and Pawelek 2009).

\section{Methodology used for investigating changes in melanoma signaling pathways}

Taking into account that most metabolism and signaling pathways are connected (Fig. 1), the reprogramming of one pathway may have a crucial impact on others. Therefore, the knowledge of methods allowing a detailed analysis of each pathway can help in establishing an effective treatment of several diseases including melanoma skin cancer.

\section{Analysis of energy metabolism in melanoma cells}

Glucose and glutamine uptake regulates the metabolism of a whole cell and for various tumors has been found to be significantly increased. A dynamic picture of metabolism may be explored thanks to stable isotope $\left({ }^{13} \mathrm{C}\right.$ or $\left.{ }^{14} \mathrm{C}\right)$ tracing (Allen and Young 2020). The growth medium can be supplemented with the selected concentration of $\left[\mathrm{U}_{-}{ }^{13} \mathrm{C} 6\right]$ glucose or/and $\left[\mathrm{U}-{ }^{13} \mathrm{C} 5\right]$ glutamine before adding it to the cells, to give labeling that should last for at least a couple of hours. For metastatic melanoma cell line A375, the described physiologically relevant concentration of $\left[1,2-{ }^{13} \mathrm{C}\right]$ glucose was $5 \mathrm{mM}$ (Delgado-Goñi et al. 2020). Medium samples should be collected at the start $(0 \mathrm{~h})$ and after cell treatment (e.g.,

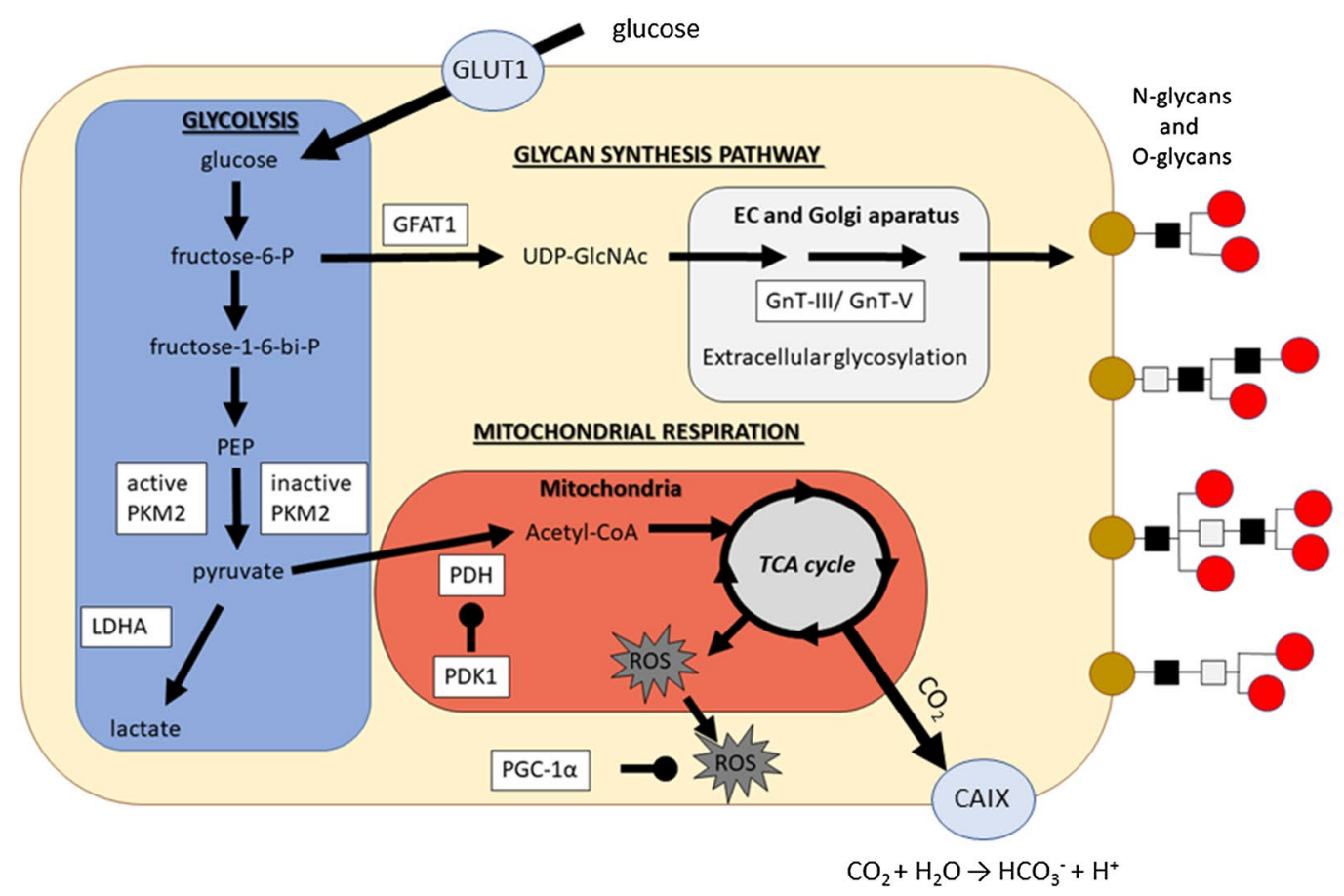

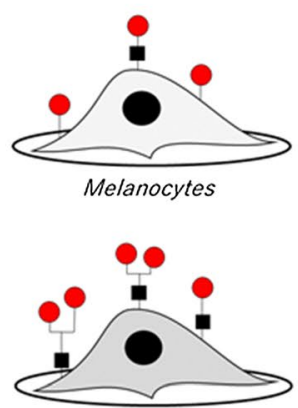

Primary melanoma

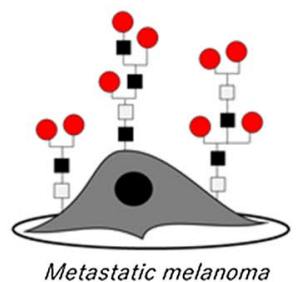

Fig. 1 The connection between various pathways in cells: glycolysis, OXPHOS, TME signaling, HBP and glycan biosynthesis pathway 
24 or $48 \mathrm{~h}$ ). Then, the cell should be collected via trypsinization, rinsed with a PBS buffer and centrifuged to obtain cell pellets, which should be stored at $-80{ }^{\circ} \mathrm{C}$. Sample preparation for gas chromatography combined with mass spectrometry (GC-MS) (Scott et al. 2011) or for nuclear magnetic resonance (NMR) spectroscopy (Wang et al. 2014; DelgadoGoñi et al. 2020) analyses is performed by the methanolchloroform extraction of the analytes like glucose, pyruvate or fatty acids from the media samples or cell pellets. A simpler analysis can be also performed with the use of commercial kits for the quantification of glucose and glutamine consumption as well as lactate production. These assays are also based on medium collection from samples and next by the addition of the appropriate enzyme (e.g., hexokinase, glucose 6-phosphate dehydrogenase, glutamate dehydrogenase, lactate dehydrogenase) provided by the manufacturer to the sample. After $30 \mathrm{~min}$ incubation time, an absorbance or fluorescence measurement is taken (Scott et al. 2011; Gkiouli et al. 2019). With such approaches, for example, a comparative profiling of metabolic fluxes in melanocytes (H3A and NEM-LP) and melanoma cell lines (WM35, Mel501, UACC903, WM793, Lu1205 and MeWo) grown under normoxic and hypoxic conditions was performed. Melanoma cells presented mainly the Warburg effect, but not all glucose was consumed during glycolysis. Glutamine was especially important for fatty acid synthesis under hypoxia (Scott et al. 2011). Furthermore, the GC-MS analysis allowed identification of up to 39 metabolites (including alcohols, amino acids organic acids, purines, pyrimidines, sugars and other metabolites) of human epidermal melanocytes (HEMn-LP) and two melanoma cell lines (A375, A2058) with a different stage of metastasis (Kim et al. 2017). The ${ }^{1}$ HNMR-based metabolomics analysis of metastatic melanoma in C57BL/6J mouse spleen showed that the selected metabolites occurrence in samples with tumor cells can be well separated from that observed with normal cells. Melanoma samples presented decreased amounts of taurine, glutamate, aspartate, $O$-phosphoethanolamine, niacinamide, ATP, lipids and glycerol derivatives; as well as increased amounts of alanine, malate, xanthine, histamine, deoxycytidine triphosphate, guanosine triphosphate, thymidine and 2'-deoxyguanosine (Wang et al. 2014).

Changes in cellular metabolism can also be observed following the gene expression and enzyme activity by qPCR or Western blot/ELISA techniques, respectively. From the cancer research point of view, some of the promising metabolic targets are proteins connected to glycolysis (GLUT1, GAPDH, PKM2, LDHA, MCT1), glutamine metabolism (xCT, GLS), fatty acids synthesis (CPT1, MGLL), pyruvate metabolism (PDK1, PDC) and other regulators (HIF, mTOR, CAIX) (Kim et al. 2006; Stubbs and Griffiths 2010; Courtnay et al. 2015; Ratnikov et al. 2017). For these analyses, cells are collected by trypsinization and RNA is obtained from cells by means of guanidinium thiocyanate-phenol-chloroform extraction (Chomzynski 1987). After quantitative determination of the RNA concentration (the absorbance read at 260/280 $\mathrm{nm}$ and 260/230 nm) and its qualitative analysis (horizontal electrophoresis in an agarose gel), reverse transcription is performed and real-time polymerase chain reactions (qPCR) performed with primers corresponding to the investigated molecular targets. In case of Western blot or ELISA techniques, the proteins are isolated from cells collected by trypsinization or mechanical scraping (depends on the molecular targets of the investigation), cell centrifugation, washing with a phosphate-buffered saline (PBS) buffer and a secondary centrifugation to obtain cell pellets, which should be stored at $-20^{\circ} \mathrm{C}$. After the protein extraction from cells, their content in samples is determined usually with the BCA Protein Assay Kit or Bradford reagent on a microplate reader. Different concentrations of bovine serum albumin (BSA) are used for the preparation of the standard curve (Eslami and Lujan 2010). Then, the samples may be provided for the electrotransfer or ELISA assay, which is followed by the incubation of the received membrane (e.g., polyvinylidene fluoride-PVDF, nitrocellulose) or multi-well plate with the blocking solution, and next with the primary and secondary antibodies (conjugated with an enzyme or fluorophore). For antibodies conjugated with enzymes, the corresponding substrate must be used. The results are obtained from the visualization of bands via absorbance/fluorescence (Western blot) or each single-well read from the whole plate (ELISA) (Andreucci et al. 2018). From such studies primary and metastatic melanoma tissues have shown an enhanced expression of GLUT1 transporter, which promoted glucose uptake and cell progression, compared to the tissue with benign nevi. Furthermore, the suppression of GLUT1 in melanoma cells with shRNA revealed significantly reduced proliferation, apoptosis resistance, migratory activity and matrix metalloproteinase 2 (MMP2) expression (Koch et al. 2015). The qPCR/Western blot investigation, performed on patient-derived melanoma cells, showed an increased HIFl $\alpha$ expression in comparison with normal melanocytes. The presence of BRAF (V600E) mutation in melanoma cells increased the expression of $H I F 1 \alpha$ as well as cell survival under hypoxic conditions (Kumar et al. 2007). In another study, three BRAF-mutant melanoma cell lines (M14, A375 and 518A2) cultured under hypoxia presented not only the overexpression of HIF $1 \alpha$, but also of CAIX (Pucciarelli et al. 2016). Melanoma cells with BRAF (V600E) mutation also suppressed the expression of the mitochondrial metabolism regulator-PGC- $1 \alpha$ (Haq et al. 2013). The transcription factor SOX2 (sex-determining region Y (SRY)-Box2) is correlated with growth, tumorigenicity as well as drug resistance and its increased expression was observed in melanoma cells. Moreover, SOX2 protein occurrence was significantly increased in A375-M6 
melanoma cells exposed to acidic medium (pH 6.7) in comparison with the neutral medium ( $\mathrm{pH}$ 7.4). This additionally induced a metabolic shift towards oxidative phosphorylation with a simultaneous slowdown of the glycolytic pathway, as confirmed by the reduced lactate production (Andreucci et al. 2018). Since hypoxia-induced changes in metabolism can affect the extracellular $\mathrm{pH}$ and oxygen consumption of cells, these parameters should be monitored, for example, in real-time by a non-invasive SDR optical sensor system (Pucciarelli et al. 2016).

On the other hand, the most advanced analysis of the in vitro cell metabolism may be performed by means of the Extracellular Flux Analyzer from Seahorse Bioscience. The XF Cell Mito Stress Kit allows the measurement of the efficiency of glycolysis and the mitochondrial oxidative phosphorylation. The measured parameters are the oxygen consumption rate (OCR) and the extracellular acidification rate (ECAR). The primary set up of the conditions required for the Seahorse experiments with different cell types includes: the appropriate cell number per well and the selection of drug concentrations necessary for the correct performance of the Seahorse analysis. The Seahorse drugs are: oligomycin — an ATP synthase inhibitor, carbonyl cyanide-4-(trifluoromethoxy) phenylhydrazone (FCCP) the protonophoric uncoupler and a mixture of Rotenone with Antimicin A (Rot/AA), which functions as the electron transport inhibitor. To enable the normalisation of the cell number in each well, one of two methods should be applied (cell counting or the total protein determination) on the plates after the Seahorse experiments (Zhang and Zhang 2019). Various melanoma cell lines (A375, ED-007, ED-013, ED-024, ED-027, ED-029, ED-034, ED-050, ED-070, ED-071, ED-117, ED-140, ED-179 and ED-196, MEL103, MEL526, and MEL697, Melmet 1, Melmet 5 and SK-MEL-28) showed higher glycolytic rates (higher basal glycolytic ECARs) compared to normal melanocytes, as well as higher maximal glycolytic capacities. In general, there were no significant differences in the basal and maximal mitochondrial respiration between melanocytes and melanoma cells (OCR) (Abildgaard et al. 2014; Bettum et al. 2015; Shankar Babu et al. 2018).

The natural by-products of cell metabolism, like reactive oxygen (ROS) and nitrogen (RNS) species, play significant roles in cell signaling and homeostasis, as well as allowing measurement of changes in metabolism (Ozcan and Ogun 2015; Ciccarese and Ciminale 2017). Accumulation of ROS in cells has been recognized as one of the mechanisms leading to DNA damage associated with mitochondrial dysfunction and apoptosis (Kwon et al. 2019). To detect ROS and RNS several analytical approaches have been used, including electron paramagnetic resonance (EPR), chemiluminescence and fluorescence. For example, measurement of the intracellular and mitochondrial superoxide $\left(\mathrm{O}_{2}^{-{ }^{-}}\right)$can be performed using hydroethidine (HE) and Mito-SOX. For establishing the amount of the released peroxynitrite $\left(\mathrm{ONOO}^{-}\right)$the most frequently used probe is dihydrorhodamine (DHR). Dichlorodihydrofluorescein diacetate (DCFH-DA) may be used for the determination of intracellular hydrogen peroxide $\left(\mathrm{H}_{2} \mathrm{O}_{2}\right)$, but this assay has several limitations and artifacts associated with the experiment (more details in Kalyanaraman et al. 2012). The optimization of these assays is connected with the selection of the proper number of cells per well and also the concentration of the labeling marker for each cell line individually. As positive controls extracellular $\mathrm{H}_{2} \mathrm{O}_{2}$ or tertbutyl hydroperoxide ( $t$-BHP) may be used in a cell-dependent manner, and to reduce the amount of reactive species production GSH may be applied (Slamenova et al. 2013; Kwon et al. 2019). The capability of suppressing increased ROS levels was confirmed only in melanocytes. Therefore, the increased ROS level occurrence in melanoma cells has been confirmed so far through multiple mechanisms (de Melo et al. 2013; Liu-Smith et al. 2014).

An important issue is also the metabolic plasticity of cancer cells (Avagliano et al. 2020; Rodríguez-Enríquez et al. 2020). Atomic force microscopy (AFM) in the force spectroscopy mode is widely used for measuring mechanical properties of cells in their physiological conditions in vitro. From the AFM measurements, force-distance curves are analyzed and the Young's modulus (in some cases called elastic modulus) is typically calculated using the Hertz-Sneddon model, which includes the geometry of the tip (Lekka 2017). Mechanical properties of cells mainly correspond to the organization of the cell cytoskeleton, especially actin filaments at the indentation depth up to $500 \mathrm{~nm}$ (Pogoda et al. 2012; Gostek et al. 2015). In case of melanoma cells, various research has shown significant differences between normal cells and malignant cells, where normal cells obtain higher values of cells' elastic values (Lekka et al. 1999, 2012). This is also the issue for melanocytes and melanoma cells (Sobiepanek et al. 2017); however, cell stiffness is a dynamic property of the primary and metastatic melanoma cell lines, thus the ability to vary their degree of elasticity towards both very low and very high values is a marker of malignancy (Weder et al. 2014). Therefore, changes in melanoma cell stiffness cannot be interpreted in a simple way and the use of AFM analysis for diagnostic purposes in case of patients with melanoma should be approached with extreme caution. Yet, some attempts have also been made to relate the dependence of melanin load of cells with the mechanical properties of melanoma cells (Sarna et al. 2018). The presence of melanin granules dramatically modified the elastic properties of the pigmented melanoma cells, where the non-pigmented cells presented the lowest values of Young's modulus (ca. $2 \mathrm{kPa}$ ) and heavily pigmented the highest values (ca. $7 \mathrm{kPa}$ ) (Sarna et al. 2014). On the other hand, melanoma cells containing melanin were less capable of spread in mice than cells 
without the pigment, which indicates that the presence of melanin inhibits melanoma metastasis (Sarna et al. 2019). The melanin content in cells could also be determined by electron paramagnetic resonance (EPR) spectroscopy based on the intensity and spectral parameters of characteristic EPR signals of eumelanin and pheomelanin (Sarna et al. 2014). Using a single scan with EPR the effect of free radicals in melanin and melanoma cells can be obtained (Pilawa et al. 2017; Żądło 2019). The ability of EPR imaging to accurately map melanoma depends on the concentration of melanin in the sample, which is proportional to the growth stage of the tumor (Godechal et al. 2012).

Finally, an indirect analysis to study changes in cell metabolism is conducted by means of cell migration tests. For this purpose, a transwell migration/invasion assay, wound-healing assay or individual cell-tracking systems may be utilized. Although the inserts for the transwell migration/ invasion assays are quite expensive, the test brings further knowledge of cell behavior than the simple wound-healing assay. Concerning the creation of the wound in the cell culture, it can be done manually using a pipette tip (in this case it is called the scratch assay) or first a silicon insert may be placed in the culture dish and then the suspension of cells is added. Contrary to other tests, the individual celltracking requires cell seeding at low density. Furthermore, the analysis requires the use of a special type of microscope equipped with a cage incubator (at $37{ }^{\circ} \mathrm{C}$ and $5 \% \mathrm{CO}_{2}$ ) and performing time-lapse imaging to collect a large amount of data for analysis. Melanoma cells feature a highly migratory phenotype facilitating the colonization of distant organs like lung, liver, spleen or brain (Zhu et al. 2004; Pucciarelli et al. 2016; Pijuan et al. 2019). On the other hand, cell migration is also strongly influenced by the glycosylation of cells.

\section{Analysis of melanoma cell glycosylation}

Changes in the glycosylation pattern of surface glycans lead to the preferential occurrence of certain types of glycoconjugates in several diseases including cancer. Within several years many glycosylation-related disorders have been identified and assigned to a proper class of glycans ( $\mathrm{N}$-linked, glycosaminoglycan, dystroglycanopathy, $O$-GalNAc/O-GlcNAc, GPI-anchor, glycolipid, $O$-fucose $/ O$-glucose) (Freeze et al. 2014). These differences appear mainly due to changes in glycosyltransferases and glycosidases, which regulate the glycan homeostasis (Taniguchi and Kizuka 2015). Similarities and differences in the carbohydrate chains of $N$ - and $\mathrm{O}$-glycans present on the cell surface are specific for particular phases of cancer development. Depending on the glycosylation changes, the following processes can have different advances: adhesion to endothelium, cell-cell adhesion, cell motility, immune system modulation, growth factor modulation and growth receptor regulation (Potapenko et al.
2010). Thus, one of the options for investigating the possible changes occurring in the glycosylation of cells is by means of the expression of genes encoding enzymes responsible for the glycan biosynthesis pathway or by checking enzyme activity. However, because glycan biosynthesis is a complex process, which consists of several steps to receive the final form of $\mathrm{N}$-glycans or $\mathrm{O}$-glycans with side chains, the selection of the gene/enzyme should be made with caution (Vasconcelos-dos-Santos et al. 2015). Some of the crucial enzymes are glutamine fructose-6-phosphate aminotransferase (GFAT) and dolichyl-phosphate mannosyl transferase (also known as dolichol-phosphate mannose synthase, DPMs). Glutamine fructose-6-phosphate aminotransferase-1 (GFAT-1) is one of the two main isoforms of the GFAT family, which converts fructose 6-phosphate to glucosamine 6-phosphate and it is the rate-limiting enzyme of the HBP pathway (Gélinas et al. 2018). Dolichyl-Phosphate Mannosyl transferase Subunit 1 (DPM1) is the largest subunit of the DPMs, which represents the catalytic center of the enzyme that generates dolichol phosphate-mannose (Dol-P-Man) from GDP-mannose (GDP-Man) and dolichol phosphate (Dol-P) (Aebi and Hennet 2001). These two enzymes are essential for the first few steps of glycan biosynthesis; thus, a differential expression of genes encoding them may show differences in the degree of cell glycosylation as well as a possible switch of glucose metabolism to the HBP pathway. Furthermore, the skin cutaneous melanoma has shown the upregulation of the HBP pathway activity as well as single HBP components in comparison with the normal skin (Jia et al. 2020). On the other hand, the most important enzymes responsible for the $\mathrm{N}$-glycan branching are GnT-III (encoded by gene MGAT3), GnT-IV (encoded be two genes MGAT4a and MGAT4b), GnT-V (encoded by gene MGAT5), GnTIX (encoded by gene $M G A T 9$ ), Fut8 (encoded by gene FUT8) and GCNT2 (Taniguchi and Kizuka 2015; Harada et al. 2019; Dimitroff 2019). The overexpression of MGAT3 and MGAT5 genes were identified in metastatic melanoma cell line WM266-4 isolated from the lymph node (Bubka et al. 2014; Link-Lenczowski et al. 2018). The primary and metastatic melanoma specimens present a strong inverse relationship between GCNT2 expression and metastases. With increasing metastatic potential, cells mostly lacked the I-branched $\mathrm{N}$-glycan antennae which was due to the depressed GCNT2 expression (Sweeney et al. 2018). In general, the results obtained for melanoma cells demonstrate that the core structure profile of $\mathrm{N}$-glycans may be inherited at the genetic level during the multiple selection cycles of B16 variants (B16-F1, B16-F10, B16-BL6) (Harada et al. 2019).

On the other hand, the carbohydrate structures may be detected by lectins, proteins or glycoproteins of a non-immunological origin, commonly found in viruses, bacteria, fungi, plants, animals and humans. Their characteristic feature 
is the ability to bind specifically and reversibly distinct mono- and oligosaccharides without the use of enzymes. The lectin-carbohydrate binding is obtained through noncovalent interactions like hydrogen bonds, van der Waals forces and hydrophobic interactions. Binding with carbohydrates located on the cells' surface ensures the occurrence of the connection between cells (Coelho et al. 2017). Due to the multivalent nature of these interactions, lectins have a high affinity for the spatial structures of oligosaccharides (Yakovleva et al. 2009). Unlike most glycoproteins, due to the presence of at least two binding sites, they are capable of agglutinating or precipitating glycoconjugates (Peumans and Van Damme 1995). Some of the typically used lectins are: concanavalin A (Con A; specific for $\alpha$-D-mannose and $\alpha$-D-glucose, Helix pomatia agglutinin (HPA; $N$-acetylgalactosamine), Peanut agglutinin (PNA; $\beta$-galactose), Ricinus communis agglutinin (RCA; $\beta$-D-galactose), Soybean agglutinin (SBA; galactose $/ \alpha$ - and $\beta$ - $N$-acetylgalactosamine), Ulex europaeus agglutinin-I (UEA-I; L-fucose) and Wheat germ agglutinin (WGA, $N$-acetylglucosamine) (Wu et al. 2008; Sobiepanek 2017).

Some studies using lectins to detect specific sugars have shown that the detection of altered glycoconjugates can help to distinguish abnormal cells from normal ones (Laidler et al. 2006). To study this characteristic effect, techniques like Western blotting (Przybyło et al. 2002; Hoja-Łukowicz et al. 2014), fluorescence microscopy (Peiris et al. 2012), enzyme-linked lectin sorbent assays (ELLSA) (Wu et al. 2008), structural studies by X-ray crystallography and nuclear magnetic resonance (NMR) (Henrichsen et al. 2004), high-performance liquid chromatography (HPLC) (Taniguchi and Kizuka 2015), affinity chromatography (HPLAC), affinity capillary electrophoresis (ACE) (Lebed et al. 2007), electrochemical impedance spectroscopy (EIS) (Carvalho et al. 2014) and several mass spectrometry techniques (ICPMS, MALDI-MS, LC-MS/MS) (Ciołczyk-Wierzbicka et al. 2004; Gao et al. 2016; Zhou et al. 2017) may be used. Although highly informative, these methods are mostly destructive for the samples; likewise, they require expensive and time-consuming preparations (Sobiepanek 2017). For example, the structural analysis of glycans present on the melanoma cell surface by means of MALDI-TOF-MS is achieved by: cell harvesting, homogenization via sonication, immunoprecipitation, electrotransfer, deglycosylation, sugar extraction, esterification of sialic acid, microcolumn cleanup of sugars, recording of the matrix-assisted laser description ionisation mass spectra and finally detailed data analysis with some basic assumptions for these kinds of results (e.g., specified algorithms). This approach has provided interesting insights concerning the glycosylation pattern of N-cadherins on melanoma cells. Cells from the primary tumor site (WM35-RGP) possessed high-mannose and biantennary complex type glycans with $\alpha 2-6$ linked sialic acid, whereas different metastatic cell lines (WM239-skin, WM9-the lymph nodes and A375-solid tumor) possessed mostly tri- or tetra-antennary complex type glycans. Furthermore, $\mathrm{N}$-cadherin in some metastatic cell lines contained heavily $\alpha$-fucosylated complex type chains with $\alpha 2,3$ linked sialic acid (Ciołczyk-Wierzbicka et al. 2004). However, during the MS analysis some monosaccharides may be removed from the glycan structure due to the weakness of the glycosidic bonds, thus the structures inferred may not be identical with the real ones (Zhou et al 2017). $N$-Glycans may be also released from cells by PNGase F digestion, and then fluorescently labelled and separated by anion-exchange HPLC. Glycans may be analysed by reverse-phase HPLC or MS to determine their structure. The relative amounts of complextype, hybrid-type and high-mannose-type glycans in melanoma cells B16-F10 were found to be $62 \%, 1 \%$ and $37 \%$, respectively (Harada et al. 2019).

Moreover, label-free nanotechnologies such as atomic force microscopy (AFM), quartz crystal microbalance (QCM) and quartz crystal microbalance with dissipation energy monitoring (QCM-D) (Lebed et al. 2007; Pei et al. 2012; Sobiepanek et al. 2017), surface plasmon resonance (SPR) or isothermal titration calorimetry (ITC) (Li et al. 2015) are also increasingly gaining attention in the study of interactions between lectins and glycans on cells. They enable not only the detection of the selected glycoconjugates, but also the determination of the kinetic and thermodynamic parameters of the interactions occurring as well as their affinity. Some methods require not only the isolation of glycans, but also purification of the selected target (for SPR and ITC); whereas others can use whole cells with glycan structures present on their membranes as targets (AFM and QCM/QCM-D). The integral membrane proteins require a lipid bilayer environment to maintain their structure and function, thus methods which do not require the isolation of glycoconjugates from cells give more reliable results imitating a biologically relevant environment for these molecules. Furthermore, to obtain the appropriate concentration of glycans in solution, the tissue dimension must be of a sufficient size otherwise the SPR and ITC measurements will not be possible (Katrlík et al. 2010; Li et al. 2015; Sobiepanek and Kobiela 2018). In AFM microscopy, the lectin must be immobilized on the AFM tip and during the measurement it interacts with the glycans present on a single cell (Fig. 2a), but during QCM/QCM-D measurements the lectin is introduced in the buffer solution from where it can directly bind with specific glycans present on all cells cultured on the surface of the sensor (Fig. 2b) (Sobiepanek et al. 2017). In general, the optimization of these measurements is concentrated on selecting: the proper buffer, type of surface for cell growth and the number of cells cultured on these surfaces (Sobiepanek and Kobiela 2021). A properly established measurement protocol allows one to obtain information about the occurring lectinglycan interactions. For example, the glycosylation level on 
$\mathbf{A}$

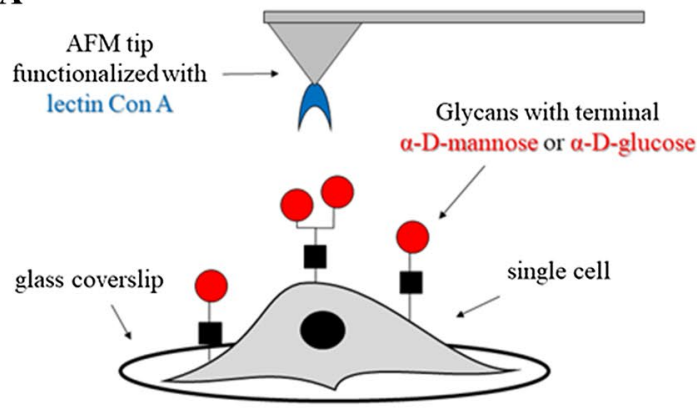

B

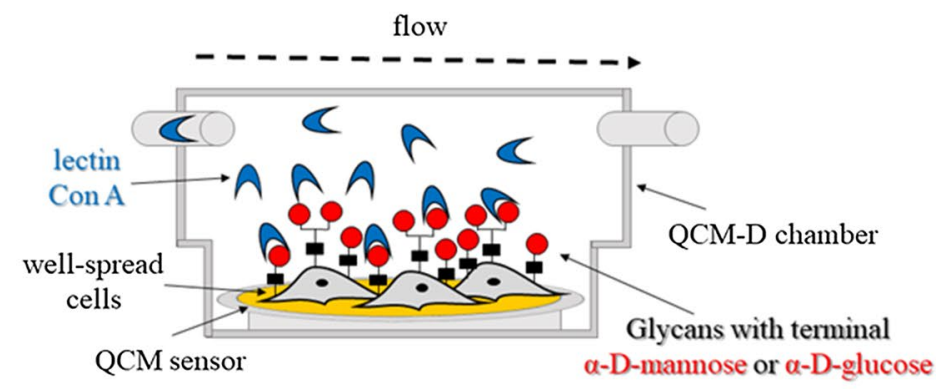

C

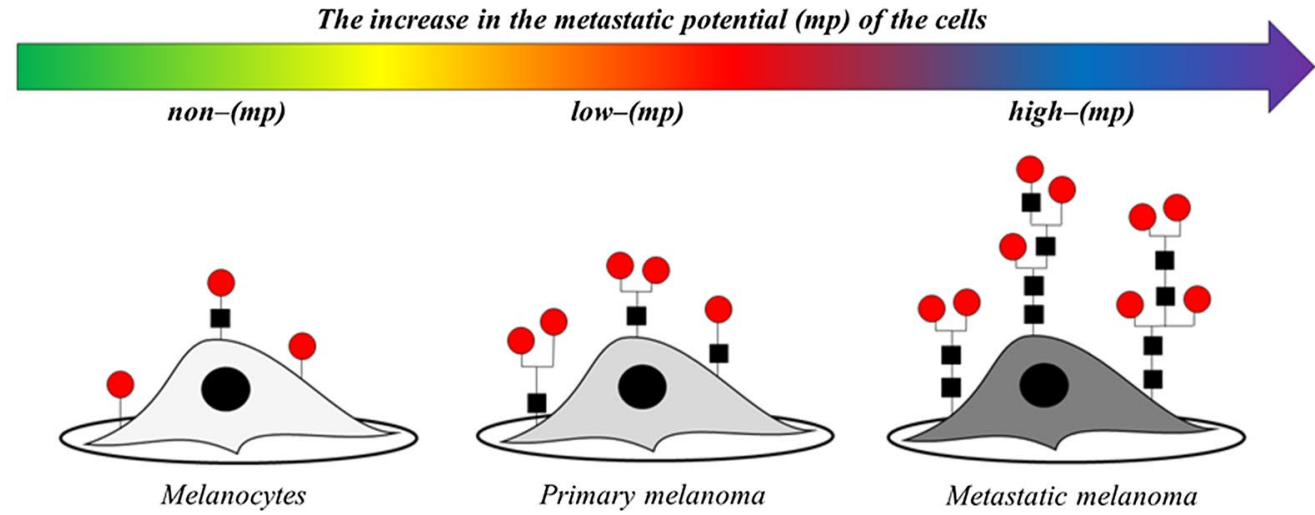

Fig. 2 Biophysical characterization of glycans present on melanocytes/melanoma cells by means of label-free methods: AFM after tip functionalization with lectin (a) and QCM-D during the measurement with lectin (b). Schematic representation of the distinction of mela- noma cells with a higher metastatic potential from the cells with a lower metastatic potential based on the performed measurements of lectin binding to glycans on cells by means of AFM/QCM-D (c)

requesting approval of the Food and Drug Administration (FDA). Several groups of compounds may be mentioned in case of melanoma reprogramming treatment beginning with inhibitors of various kinases, through to effectors of metabolism, and also by using endogenous as well as exogenous molecules.

\section{Kinase inhibitors}

Metabolic dysregulation in melanoma is modulated by oncogenic activation. The most common genetic alterations are activating mutations in the BRAF proto-oncogene. It is described that inhibition of BRAF kinase leads to a decrease in glucose uptake both in cell culture and in melanoma patients (Haq 2014; Rankin et al. 2016; Wigerup et al. 2016). Thus, the adaptive response to BRAF/MEK inhibitors (BRAFi/MEKi) is associated with the shift from glycolysis toward oxidative phosphorylation (Avagliano et al. 2020).

One of the best-known inhibitors of the BRAF kinase is Vemurafenib (VEM, PLX4032) — approved in 2011 by FDA, but the majority of patients relapse within 6-7 months of treatment. This is possibly due to the ability of melanoma cells to undergo reprogramming, leading to the survival of 
some tumor cells. Dabrafenib (GSK2118436) is a second best known selective BRAF-mutant inhibitor approved in 2013 (Parmenter et al. 2014; Domingues et al. 2018).

The in vitro established BRAFi-resistant clones of melanoma cells were shown to produce significantly less TCA metabolism-related metabolites (acetate, fumarate), PPP metabolites (ribose), glutamine (glutamine, glutamate, glutathione) and HBP metabolites (UDP-GlcNAC) by NMR metabolic analysis. At the same time, it was shown that BRAF-mutant melanoma cells, which were sensitive to BRAF inhibitors, presented higher glycolytic, bioenergetic and phospholipid metabolic activity compared to resistant cells. Also, the $\left[1,2-{ }^{13} \mathrm{C}\right]$ glucose administration revealed that BRAFi-sensitive cells had a significantly higher glucose uptake in comparison with drug resistant cells (DelgadoGoñi et al. 2020).

Gene expression and protein level/enzyme activity analysis brought a great deal of information about the influence of vemurafenib on different melanoma cells. Normally, melanoma cells with activation of the BRAF/MAPK pathway (M14, MALME-3M, UACC-62, UACC257) presented suppressed levels of MITF and $P G C-1 \alpha$ - the master regulator of the mitochondrial biogenesis and OXPHOS. However, after cell treatment with VEM, $P G C$ - $1 \alpha$ expression was induced even up to 3-14 fold. Nevertheless, VEM had no influence on $P G C$ - $1 \alpha$ gene expression in case of wildtype melanoma cells (MeWo) treatment (Haq et al. 2013). Vemurafenib-treated A375 cells under hypoxic conditions also exhibited an enhanced cell proliferation rate and migratory capacity compared to the normoxic vemurafenib-treated A375 cells. The expression of HIF $1 \alpha$ and CAIX were reduced in vemurafenib-treated M14 and 518A2 cells, but not in A375 cells (all three cell lines are BRAF-mutants) (Pucciarelli et al. 2016). BRAF-mutant melanoma cells (A375) also presented the induced expression of GLUT-1, which in line enhanced glucose transport into these cells (Delgado-Goñi et al. 2020). The inhibition of the BRAFmutant cells by the VEM treatment suppressed glycolysis independently of cell cycle progression and cell death via the suppression of the expression of HK2 as well as glucose transporter-1 and -3 (GLUT1/3) (Parmenter et al. 2014). But when establishing VEM-resistant clones of the BRAFmutant cell lines, they not only expressed reduced levels of GLUT-1 (Delgado-Goñi et al. 2020), but also the glucose metabolism was restored (Parmenter et al. 2014).

\section{Effectors of metabolism}

Nowadays, a popular therapeutic strategy for cancer treatment is to apply drugs which attack different functions of mitochondrial metabolism (bioenergetics, signaling and biosynthesis). This can change the TCA activity of cells and lead to a strong energetic stress in tumor cells inhibiting other pathways (like mTOR, PPP and HBP) and inducing autophagy (Missiroli et al. 2020). But the reprogramming of tumor cell metabolism is a very complex and heterogeneous process, which is influenced by a wide variety of genetic and non-genetic strategies (Abildgaard et al. 2014). Most of the available drugs are inhibitors of the electron transport chain complexes in mitochondria (complex I inhibitors-Metformin, BAY 87-2243, IACS-010,759, MitoTam, complex II inhibitor-Lonidamine; general ECT inhibitor-VLX600) or inhibitors of the selected enzymes (PDH inhibitors-CPI613 and dichloroacetate; GAPDH inhibitor-iodoacetate; PK inhibitor-lonidamine and lapachol; LDHA inhibitorgossypol) (Hosseini et al. 2017; Missiroli et al. 2020). On the other hand, combination therapies involving metabolic effectors with an immune checkpoint blockade (ICB), chemotherapy, radiation and/or a diet may be even more effective than monotherapy, but only when the influence of each drug separately is known in detail (Bader et al. 2020).

Pyruvate dehydrogenase promotes the flux of carbohydrates into mitochondria and enhances aerobic oxidation of glucose. The known PDH cofactors are $\alpha$-lipoic acid and ATP citrate lyase (ACL). Competitive treatment of B16-F10 melanoma cells with these compounds as well as with the hydroxycitrate inhibitor resulted in a significant cell growth inhibition (Fischer et al. 2018). Also, pyruvate dehydrogenase kinase is an important metabolic target, given that its inhibition shifts pyruvate metabolism from lactate (glycolysis) into the TCA cycle by increasing the activity of PDH. Dichloroacetate (DCA) is a pyruvate mimetic, which decreases the lactate production presumably through the inhibition of PDK activity. This was well documented by following the changes in ECAR and OCR of Melmet 5 cells (melanoma cell line investigated by the Seahorse analyzer). A DCA-induced reduction in ECAR and an increase in OCR was observed, which was accompanied by the elevation in the ATP level (Bettum et al. 2015). This DCA-dependent shift of metabolism from glycolysis to mitochondrial oxidation reverses mitochondrial dysfunction and reactivates mitochondria-dependent apoptosis in various tumor cells. The reduction of lactate accumulation also influences strongly the acidified TME (Li et al. 2016). Furthermore, DCA potentiates the effect of vemurafenib on only BRAFmutated melanoma cells through a cooperative attenuation of energy production as it was identified by means of OCR/ ECAR measurements. In this study, DCA was tested in the concentration range of $0.5-100 \mathrm{mM}$ for up to $96 \mathrm{~h}$ and 50-100 nM VEM concentration on several melanoma cell lines. At the same time, there was no correlation between the cell response to only DCA and the expression levels of $P G C-1 \alpha$ (Abildgaard et al. 2014). Due to the fact that melanoma cells resistant to vemurafenib maintained sensitivity to DCA, this approach suggests a possible combination therapy to overcome BRAF inhibitors resistance in 
patients with melanoma (Domingues et al. 2018). On the other hand, the key mediator of glycolysis in cancer cells is pyruvate kinase. Lapachol (LAP) is an analog of shikonin, which decreases the pyruvate kinase isozyme M2 (PKM2) activity at a micromolar range. Melanoma cells treated with lapachol showed a dose-dependent inhibition of glycolysis as well as a corresponding increase in oxygen consumption, observed by means of ECAR and OCR parameters, respectively. The blockade of glycolysis by lapachol in melanoma cells also led to the decreased ATP levels and inhibition of cell proliferation (Shankar Babu et al. 2018). Another modulator of PK is lonidamine (LND), which is able to sensitize human melanoma cells to doxorubicin (DOX) chemotherapy by acidifying and de-energizing the tumor. These results were obtained by LC-MS and Seahorse analysis (Nath et al. 2018).

The matter of interest is also the involvement of the glycolytic enzyme in mitochondria induced apoptosis. Mitochondrial permeability transition pore (mPTP), a voltage and $\mathrm{Ca}^{2+}$-dependent, cyclosporine $\mathrm{A}(\mathrm{CsA})$ and ROS sensitive channel in the inner mitochondrial membrane, is a key effector in the intrinsic mitochondrial apoptotic pathways and necrosis (Bonora et al. 2020). The MPTP is formed at the interface between two mitochondrial ATP synthase (F-ATP) dimers (Nesci 2018). Various adaptive mechanisms of tumor cells that desensitize the mPTP to $\mathrm{Ca}^{2+}$ and ROS, thereby playing an important role in the resistance of tumors to apoptosis, have already been described (Bernardi et al. 2015). The $\mathrm{Ca}^{2+}$ - and ROS-dependent signaling pathways affecting transition of the F-ATP synthase from an energyconserving to an energy-dissipating mechanism open new perspectives for therapeutic intervention aimed at the mPTP induction in cancer cells. Many used and potential chemotherapeutics that induce the mPTP, mainly by oxidative stress, are under investigation (Rasola and Bernardi 2014). Particularly interesting is isoform II of hexokinase (HK II), the first enzyme of glucose metabolism bound to the outer mitochondrial membrane at mitochondria-endoplasmic reticulum contact sites that is predominantly expressed in malignant cells and has implications for tumor progression. The detachment of HK II from mitochondria induces mPTP opening and the subsequent apoptosis in several tumor cell models (Chiara et al. 2008; Ciscato et al. 2020). Substances triggering this process are being extensively researched (Bonora et al. 2020).

\section{Other reprogramming compounds}

The endocannabinoid system (ECS), which consists of cannabinoid receptors, endogenous cannabinoids (endocannabinoids like $\mathrm{N}$-arachidonylethanolamine, named anandamide) and enzymes responsible for the synthesis, transport and degradation of endocannabinoids, plays a dual role in tumorigenesis, the inhibition of tumor growth and metastatic spread (Moreno et al. 2018; Ramer et al. 2019). From a therapeutic point of view, the association of ECS with glucose and lipid metabolism is very interesting and some endocannabinoids are deeply involved in the maintenance of energy balance (Kim et al. 2011; Zaccagnino et al. 2011; Nava-Molina et al. 2020). Anandamide (AEA) has a confirmed action on the disturbance of the glucose uptake in skeletal muscle cells (Eckardt et al. 2009), thus one can assume that changes in the glucose distribution may affect general cell metabolism including the aberrant glycosylation of cells. This result may be observed due to the existing direct connection between glycolysis and the HBP pathway (Vasconcelos-dos-Santos et al. 2015). Moreover, AEA disturbs mitochondrial bioenergetics by the inhibition of oxidative phosphorylation in isolated mitochondria from rat liver (Zaccagnino et al. 2011).

Some antibiotics may also present a significant influence on cell metabolism by inducing mitochondrial dysfunction and oxidative damage in mammalian cells. Taking this into consideration, cell culture studies on metabolism should probably be done in an antibiotic free media (Elliott and Jiang 2019). An example is the class of aminoglycoside antibiotics, which consists mainly of gentamicin and geneticin. They show diverse usability against prokaryotic and eukaryotic cells, especially due to the fact that cell response depends on its metabolism (Sobiepanek et al. 2020c). The addition of gentamicin in cell culture media inhibits: the mitochondrial membrane potential gradient in the cells, the upregulation of HIFla gene expression, the glycolytic enzymes and glucose transporters as well as the increase of lactate production, induction of mitochondrial superoxide and the increase in the DNA oxidative damage in the cells (Elliott and Jiang 2019). The addition of geneticin (G418) may cause the change of glucose flux from glycolysis towards either the TCA cycle or other biosynthetic pathways in various cell lines like baby hamster kidney (BHK) and Chinese hamster ovary (CHO) cells (Yallop and Svendsen 2001; Yallop et al. 2003).

On the other hand, there are various inhibitors of cell glycosylation, which can interrupt the biosynthesis of the complex type glycans. This generally decreases cell migration, adhesion and angiogenesis. Some of the well-known inhibitors of glycosidases are plant alkaloids like swainsonine (SW, $\alpha$-mannosidase II and lysosomal $\alpha$-mannosidase I inhibitor), deoxymannojirimycin (DMM, $\alpha$-mannosidase I inhibitor), deoxynojirmycin (DNJ, glycosidase I), castanospermine (CS, glycosidase I and II inhibitor) (Powell 2001; Ciołczyk-Wierzbicka et al. 2004; Wojtowicz et al. 2012). Similarly interesting are sugar analogues like 2-deoxyglucose (2-DG) or 2-NBDG, which block glycotransferases and form dolichol-P-2-deoxyglucose that cannot be further extended. 2-DG has a further impact on the gene expression, 
protein phosphorylation of the signalling pathway, blocking the cell cycle progression, DNA repair and cell apoptosis (Wojtowicz et al. 2012; Lucena et al. 2016). Furthermore, 2-DG and 2-fluoro-deoxyglucose (2-FDG) are also the inhibitors of glycolysis, which compete for the HK binding (a key glycolytic enzyme) (Vasconcelos-dos-Santos et al. 2015; Missiroli et al. 2020).

\section{Conclusions}

Melanoma is a highly heterogenous tumor, thus a combined therapy targeted at various metabolic pathways is needed to overcome the problem that many patients develop resistance to monotherapy. Novel factors able to remodel cell metabolism must be first analyzed separately to then be used in combined therapies. Various kinases and effectors of metabolism are currently the most promising drug target combination in therapy against melanoma. A detailed knowledge of each metabolic pathway is of great importance while analyzing the influence of new remodeling factors. This includes specific enzymes, transcription factors as well as proteins or glycoproteins. The most important pathways are glycolysis, TCA cycle, glutamine pathway, PPP pathway, HBP connected with the glycan biosynthesis pathway and in case of melanoma MAPK and mTOR pathways. For investigating various changes of specific markers and physical properties during melanoma development from nevus to metastases different types of analysis are highly recommended. These analyses include qPCR, Western blot/ ELISA techniques, NMR, GC/LC-MS, Seahorse analysis as well as various label-free techniques (AFM, ITC, SPR, QCM/QCM-D).

Acknowledgements This work was supported by the Warsaw University of Technology (Poland), the Sapienza University of Rome (Italy), Grants no. RG11816430AF48E1, RM11916B46D48441, RP11715C644A5CCE, GA116154C8A94E3D and the Grant from Associazione Italiana Ricerca sul Cancro (AIRC) under IG 2019ID. 23125 project (FC), the National Science Centre (Poland) Grants no. 2017/27/N/ST4/01389 and 2018/28/T/ST4/00511 (AS), BIOTECHMED-1 project granted by the Warsaw University of Technology under the program Excellence Initiative: Research University (ID-UB) (TK) as well as the Cost Action CA15126 ARBRE-MOBIEU for the STSM no. 44705, European Cooperation in Science and Technology (ECOST-STSM-Request-CA15126-44705).

Open Access This article is licensed under a Creative Commons Attribution 4.0 International License, which permits use, sharing, adaptation, distribution and reproduction in any medium or format, as long as you give appropriate credit to the original author(s) and the source, provide a link to the Creative Commons licence, and indicate if changes were made. The images or other third party material in this article are included in the article's Creative Commons licence, unless indicated otherwise in a credit line to the material. If material is not included in the article's Creative Commons licence and your intended use is not permitted by statutory regulation or exceeds the permitted use, you will need to obtain permission directly from the copyright holder. To view a copy of this licence, visit http://creativecommons.org/licenses/by/4.0/.

\section{References}

Abildgaard C et al (2014) Bioenergetic modulation with dichloroacetate reduces the growth of melanoma cells and potentiates their response to BRAFV600E inhibition. J Transl Med 12(1):247. https://doi.org/10.1186/s12967-014-0247-5

Aebi M, Hennet T (2001) Congenital disorders of glycosylation: genetic model systems lead the way. Trends Cell Biol 11(3):136-141. https://doi.org/10.1016/S0962-8924(01) 01925-0

Akella NM, Ciraku L, Reginato MJ (2019) Fueling the fire: emerging role of the hexosamine biosynthetic pathway in cancer. BMC Biol 17(1):52. https://doi.org/10.1186/s12915-019-0671-3

Allen DK, Young JD (2020) Tracing metabolic flux through time and space with isotope labeling experiments. Curr Opin Biotechnol 64:92-100. https://doi.org/10.1016/j.copbio.2019.11.003

Amelio I et al (2014) Serine and glycine metabolism in cancer. Trends Biochem Sci 39(4):191-198. https://doi.org/10.1016/j.tibs.2014. 02.004

Ando $\mathrm{H}$ et al (2012) Melanosomes are transferred from melanocytes to keratinocytes through the processes of packaging, release, uptake, and dispersion. J Investig Dermatol 132(4):1222-1229. https://doi.org/10.1038/jid.2011.413

Andreucci E et al (2018) SOX2 as a novel contributor of oxidative metabolism in melanoma cells. Cell Commun Signal 16(1):87. https://doi.org/10.1186/s12964-018-0297-z

Austin S, St-Pierre J (2012) PGC1 and mitochondrial metabolismemerging concepts and relevance in ageing and neurodegenerative disorders. J Cell Sci 125(21):4963-4971. https://doi.org/10. $1242 /$ jcs. 113662

Avagliano A et al (2020) Metabolic plasticity of melanoma cells and their crosstalk with tumor microenvironment. Front Oncol 10:722. https://doi.org/10.3389/fonc. 2020.00722

Bader JE, Voss K, Rathmell JC (2020) Targeting metabolism to improve the tumor microenvironment for cancer immunotherapy. Mol Cell 78(6):1019-1033. https://doi.org/10.1016/j.molcel. 2020.05.034

Bernardi $\mathrm{P}$ et al (2015) The mitochondrial permeability transition pore: channel formation by F-ATP synthase, integration in signal transduction, and role in pathophysiology. Physiol Rev 95(4):11111155. https://doi.org/10.1152/physrev.00001.2015

Bettum IJ et al (2015) Metabolic reprogramming supports the invasive phenotype in malignant melanoma. Cancer Lett 366(1):71-83. https://doi.org/10.1016/j.canlet.2015.06.006

Boiko AD et al (2010) Human melanoma-initiating cells express neural crest nerve growth factor receptor CD271. Nature 466(7302):133-137. https://doi.org/10.1038/nature09161

Bonora M et al (2020) Physiopathology of the permeability transition pore: molecular mechanisms in human pathology. Biomolecules 10(7):998. https://doi.org/10.3390/biom10070998

Bubka M et al (2014) 'Overexpression of $N$-acetylglucosaminyltransferases III and V in human melanoma cells. Implications for MCAM N-glycosylation. Biochimie 103:37-49. https://doi.org/ 10.1016/j.biochi.2014.04.003

Carvalho F et al (2014) Evaluating the equilibrium association constant between ArtinM Lectin and myeloid leukemia cells by impedimetric and piezoelectric label free approaches. Biosensors 4(4):358-369. https://doi.org/10.3390/bios4040358

Chiara $\mathrm{F}$ et al (2008) Hexokinase II detachment from mitochondria triggers apoptosis through the permeability transition pore 
independent of voltage-dependent anion channels. PLoS One 3(3):e1852. https://doi.org/10.1371/journal.pone.0001852

Chomzynski P (1987) Single-step method of RNA isolation by acid guanidinium thiocyanate-phenol-chloroform extraction. Anal Biochem 162(1):156-159. https://doi.org/10.1006/abio.1987. 9999

Christiansen MN et al (2014) Cell surface protein glycosylation in cancer. Proteomics 14(4-5):525-546. https://doi.org/10.1002/ pmic. 201300387

Ciccarese F, Ciminale V (2017) Escaping death: mitochondrial redox homeostasis in cancer cells. Front Oncol 7:117. https://doi.org/ $10.3389 /$ fonc. 2017.00117

Ciołczyk-Wierzbicka D et al (2004) The structure of the oligosaccharides of $\mathrm{N}$-cadherin from human melanoma cell lines. Glycoconj J 20(7/8):483-492. https://doi.org/10.1023/B:GLYC.00000 38294.72088.b0

Ciscato $\mathrm{F}$ et al (2020) Hexokinase 2 displacement from mitochondriaassociated membranes prompts $\mathrm{Ca}^{2+}$-dependent death of cancer cells. EMBO Rep. https://doi.org/10.15252/embr.201949117

Coelho LCBB et al (2017) Lectins, interconnecting proteins with biotechnological/pharmacological and therapeutic applications. Evid Based Complement Altern Med 2017:1-22. https://doi. org/10.1155/2017/1594074

Courtnay R et al (2015) Cancer metabolism and the Warburg effect: the role of HIF-1 and PI3K. Mol Biol Rep 42(4):841-851. https:// doi.org/10.1007/s11033-015-3858-x

Dall'Olio F (2012) Mechanisms of cancer-associated glycosylation changes. Front Biosci 17(1):670. https://doi.org/10.2741/3951

de Melo FHM, Molognoni F, Galvonas M (2013) The role of oxidative stress in melanoma development, progression and treatment. In: Davids L (ed) Recent advances in the biology, therapy and management of melanoma. InTech. https://doi.org/10.5772/54937

Dehdashtian A et al (2018) Anatomy and physiology of the skin. In: Riker AI (ed) Melanoma: a modern multidisciplinary approach. Springer International Publishing, Cham, pp 15-26. https://doi. org/10.1007/978-3-319-78310-9_2

Delgado-Goñi T et al (2020) Increased inflammatory lipid metabolism and anaplerotic mitochondrial activation follow acquired resistance to vemurafenib in BRAF-mutant melanoma cells. Br J Cancer 122(1):72-81. https://doi.org/10.1038/s41416-019-0628-x

Dimitroff CJ (2019) I-branched carbohydrates as emerging effectors of malignant progression. Proc Natl Acad Sci 116(28):13729_ 13737. https://doi.org/10.1073/pnas.1900268116

Domingues B et al (2018) Melanoma treatment in review. Immunotargets Ther 7:35-49. https://doi.org/10.2147/ITT.S134842

Donoghue PCJ, Graham A, Kelsh RN (2008) The origin and evolution of the neural crest. BioEssays 30(6):530-541. https://doi.org/10. 1002/bies.20767

Dummer R et al (2016) The updated Swiss guidelines 2016 for the treatment and follow-up of cutaneous melanoma. Swiss Med Wkly 146:w14279. https://doi.org/10.4414/smw.2016.14279

Eckardt K, Sell H, Taube A, Koenen M, Platzbecker B, Cramer A, Horrighs A, Lehtonen M, Tennagels N, Eckel J (2009) Cannabinoid type 1 receptors in human skeletal muscle cells participate in the negative crosstalk between fat and muscle. Diabetologia 52(4):664-674

Elder DE (2016) Melanoma progression. Pathology 48(2):147-154. https://doi.org/10.1016/j.pathol.2015.12.002

Elliott RL, Jiang X-P (2019) The adverse effect of gentamicin on cell metabolism in three cultured mammary cell lines: "Are cell culture data skewed?" PLoS One 14(4):e0214586. https://doi.org/ 10.1371/journal.pone.0214586

Eslami A, Lujan J (2010) Western blotting: sample preparation to detection. J Vis Exp 44:2359. https://doi.org/10.3791/2359

Fenouille $\mathrm{N}$ et al (2012) The epithelial-mesenchymal transition (EMT) regulatory factor SLUG (SNAI2) is a downstream target of
SPARC and AKT in promoting melanoma cell invasion. PLoS One 7(7):e40378. https://doi.org/10.1371/journal.pone.0040378

Fischer GM et al (2018) Metabolic strategies of melanoma cells: mechanisms, interactions with the tumor microenvironment, and therapeutic implications. Pigment Cell Melanoma Res 31(1):11-30. https://doi.org/10.1111/pcmr.12661

Forman SB et al (2008) Is superficial spreading melanoma still the most common form of malignant melanoma? J Am Acad Dermatol 58(6):1013-1020. https://doi.org/10.1016/j.jaad.2007.10.650

Freeze HH et al (2014) Solving glycosylation disorders: fundamental approaches reveal complicated pathways. Am J Hum Genet 94(2):161-175. https://doi.org/10.1016/j.ajhg.2013.10.024

Gao W et al (2016) LC-MS based analysis of endogenous steroid hormones in human hair. J Steroid Biochem Mol Biol 162:92-99. https://doi.org/10.1016/j.jsbmb.2015.12.022

García-Borrón JC, Solano F (2002) Molecular anatomy of tyrosinase and its related proteins: beyond the histidine-bound metal catalytic center. Pigment Cell Res 15(3):162-173. https://doi.org/10. 1034/j.1600-0749.2002.02012.x

Gélinas R et al (2018) AMPK activation counteracts cardiac hypertrophy by reducing O-GlcNAcylation. Nat Commun 9(1):374. https://doi.org/10.1038/s41467-017-02795-4

Ghazarian H, Idoni B, Oppenheimer SB (2011) A glycobiology review: carbohydrates, lectins and implications in cancer therapeutics. Acta Histochem 113(3):236-247. https://doi.org/10.1016/j. acthis.2010.02.004

Gkiouli et al (2019) Diverse Roads Taken by 13C-glucose-derived metabolites in breast cancer cells exposed to limiting glucose and glutamine conditions. Cells 8(10):1113. https://doi.org/10. 3390/cells8101113

Godechal Q et al (2012) Optimization of electron paramagnetic resonance imaging for visualization of human skin melanoma in various stages of invasion: optimization of EPR imaging for visualization of human skin melanoma. Exp Dermatol 21(5):341-346. https://doi.org/10.1111/j.1600-0625.2012.01461.x

Gostek J et al (2015) Nano-characterization of two closely related melanoma cell lines with different metastatic potential. Eur Biophys J 44(1-2):49-55. https://doi.org/10.1007/s00249-014-1000-y

Grabacka M et al (2016a) Regulation of Ketone Body Metabolism And The Role of PPAR $\alpha$. Int J Mol Sci 17(12):2093. https://doi.org/ 10.3390/ijms17122093

Grabacka MM et al (2016b) Fenofibrate induces ketone body production in melanoma and glioblastoma cells. Front Endocrinol. https://doi.org/10.3389/fendo.2016.00005

Grabacka M et al (2017) Peroxisome proliferator-activated receptor $\alpha(\mathrm{PPAR} \alpha)$ contributes to control of melanogenesis in B16 F10 melanoma cells. Arch Dermatol Res 309(3):141-157. https://doi. org/10.1007/s00403-016-1711-2

Grabacka M, Plonka PM, Reiss K (2020) Melanoma-time to fast or time to feast? An interplay between PPARs, metabolism and immunity. Exp Dermatol 29(4):436-445. https://doi.org/10. 1111/exd.14072

Hanahan D, Weinberg RA (2011) Hallmarks of cancer: the next generation. Cell 144(5):646-674. https://doi.org/10.1016/j.cell.2011. 02.013

Haq R (2014) Metabolic dysregulation in melanoma: cause or consequence? Cancer Discov 4(4):390-391. https://doi.org/10.1158/ 2159-8290.CD-14-0173

Haq R et al (2013) Oncogenic BRAF regulates oxidative metabolism via PGC1 $\alpha$ and MITF. Cancer Cell 23(3):302-315. https://doi. org/10.1016/j.ccr.2013.02.003

Harada Y et al (2019) N-glycome inheritance from cells to extracellular vesicles in B16 melanomas. FEBS Lett 593(9):942-951. https:// doi.org/10.1002/1873-3468.13377 
Häuselmann I, Borsig L (2014) Altered tumor-cell glycosylation promotes metastasis. Front Oncol. https://doi.org/10.3389/fonc. 2014.00028

Heerboth S et al (2015) EMT and tumor metastasis. Clin Transl Med 4(1):6. https://doi.org/10.1186/s40169-015-0048-3

Henrichsen D et al (2004) Bioaffinity NMR spectroscopy: identification of an E-selectin antagonist in a substance mixture by transfer NOE. Angewandte Chemie Int Edn. https://doi.org/10.1002/ (SICI)1521-3773(19990115)38:1/2\%3c98::AID-ANIE98\%3e3.0. $\mathrm{CO} ; 2-\mathrm{V}$

Hoja-Łukowicz D et al (1861) (2017) On the trail of the glycan codes stored in cancer-related cell adhesion proteins. Biochim Biophys Acta (BBA) (General Subjects) 1:3237-3257. https://doi.org/10. 1016/j.bbagen.2016.08.007

Hoja-Łukowicz D et al (2014) The lectin-binding pattern of nucleolin and its interaction with endogenous galectin-3. Cell Mol Biol Lett. https://doi.org/10.2478/s11658-014-0206-4

Hosseini M, Kasraian Z, Rezvani HR (2017) Energy metabolism in skin cancers: a therapeutic perspective. Biochim Biophys Acta Acta (BBA) Bioenergetics 1858(8):712-722. https://doi.org/ 10.1016/j.bbabio.2017.01.013

Hwang YS et al (2019) Melanogenic effects of maclurin are mediated through the activation of cAMP/PKA/CREB and p38 MAPK/CREB signaling pathways. Oxidative Med Cell Longev 2019:1-10. https://doi.org/10.1155/2019/9827519

Jia $C$ et al (2020) GFAT1/HBP/O-GlcNAcylation axis regulates $\beta$-catenin activity to promote pancreatic cancer aggressiveness. Biomed Res Int 2020:1-13. https://doi.org/10.1155/ 2020/1921609

Kalluri R, Weinberg RA (2009) The basics of epithelial-mesenchymal transition. J Clin Investig 119(6):1420-1428. https://doi. org/10.1172/JCI39104

Kalyanaraman B et al (2012) Measuring reactive oxygen and nitrogen species with fluorescent probes: challenges and limitations. Free Radic Biol Med 52(1):1-6. https://doi.org/10.1016/j.freer adbiomed.2011.09.030

Katrlík J et al (2010) Glycan and lectin microarrays for glycomics and medicinal applications: GLYCAN AND LECTIN MICROARRAYS. Med Res Rev 30(2):394-418. https://doi.org/10. 1002/med.20195

Kim J et al (2006) HIF-1-mediated expression of pyruvate dehydrogenase kinase: a metabolic switch required for cellular adaptation to hypoxia. Cell Metab 3(3):177-185. https://doi.org/10. 1016/j.cmet.2006.02.002

Kim J, Li Y, Watkins BA (2011) Endocannabinoid signaling and energy metabolism: a target for dietary intervention. Nutrition 27(6):624-632. https://doi.org/10.1016/j.nut.2010.11.003

Kim H-Y et al (2017) Discovery of potential biomarkers in human melanoma cells with different metastatic potential by metabolic and lipidomic profiling. Sci Rep 7(1):8864. https://doi.org/10. 1038/s41598-017-08433-9

Koch A et al (2015) Glucose transporter isoform 1 expression enhances metastasis of malignant melanoma cells. Oncotarget 6(32):32748-32760. https://doi.org/10.18632/oncotarget.4977

Kumar SM et al (2007) Mutant V600E BRAF increases hypoxia inducible factor- $1 \alpha$ expression in melanoma. Can Res 67(7):3177-3184. https://doi.org/10.1158/0008-5472. CAN-06-3312

Kwon S et al (2019) Nanomedicines for reactive oxygen species mediated approach: an emerging paradigm for cancer treatment. Acc Chem Res 52(7):1771-1782. https://doi.org/10. 1021/acs.accounts.9b00136

Laidler P et al (2006) Characterization of glycosylation and adherent properties of melanoma cell lines. Cancer Immunol Immunother 55(1):112-118. https://doi.org/10.1007/ s00262-005-0019-4
Läubli H, Borsig L (2019) Altered cell adhesion and glycosylation promote cancer immune suppression and metastasis. Front Immunol 10:2120. https://doi.org/10.3389/fimmu.2019.02120

Lazova R, Pawelek JM (2009) Why do melanomas get so dark? Exp Dermatol 18(11):934-938. https://doi.org/10.1111/j.1600-0625. 2009.00933.x

Lebed K et al (2007) Atomic force microscopy and quartz crystal microbalance study of the lectin-carbohydrate interaction kinetics. Acta Phys Pol, A 111(2):273-286. https://doi.org/10.12693/ APhysPolA.111.273

Lee S-H, Griffiths JR (2020) How and why are cancers acidic? Carbonic anhydrase ix and the homeostatic control of tumour extracellular pH. Cancers 12(6):1616. https://doi.org/10.3390/cance rs 12061616

Lee J et al (2020) Geometric regulation of histone state directs melanoma reprogramming. Commun Biol 3(1):341. https://doi.org/ 10.1038/s42003-020-1067-1

Lekka M (2017) Cellular analysis by atomic force microscopy. Pan Stanford Publishing

Lekka M et al (1999) Elasticity of normal and cancerous human bladder cells studied by scanning force microscopy. Eur Biophys J 28(4):312-316. https://doi.org/10.1007/s002490050213

Lekka $\mathrm{M}$ et al (2012) Cancer cell recognition-mechanical phenotype. Micron 43(12):1259-1266. https://doi.org/10.1016/j. micron.2012.01.019

Li X et al (2015) Real-time and label-free analysis of binding thermodynamics of carbohydrate-protein interactions on unfixed cancer cell surfaces using a QCM biosensor. Sci Rep 5(1):14066. https://doi.org/10.1038/srep14066

Li B et al (2016) Dichloroacetate and metformin synergistically suppress the growth of ovarian cancer cells. Oncotarget 7(37):59458-59470. https://doi.org/10.18632/oncotarget. 10694

Link-Lenczowski P, Lityńska A (2011) Glycans in melanoma screening. Part 2. Towards the understanding of integrin N-glycosylation in melanoma. Biochem Soc Trans 39(1):374-377. https:// doi.org/10.1042/BST0390374

Link-Lenczowski P et al (2018) The glycomic effect of $N$-acetylglucosaminyltransferase III overexpression in metastatic melanoma cells. GnT-III modifies highly branched $N$-glycans. Glycoconj J 35(2):217-231. https://doi.org/10.1007/s10719-018-9814-y

Lityńska A et al (2001) Comparison of the lectin-binding pattern in different human melanoma cell lines. Melanoma Res 11(3):205212. https://doi.org/10.1097/00008390-200106000-00001

Liu-Smith F, Dellinger R, Meyskens FL (2014) Updates of reactive oxygen species in melanoma etiology and progression. Arch Biochem Biophys 563:51-55. https://doi.org/10.1016/j.abb. 2014.04.007

Lucena MC et al (2016) Epithelial mesenchymal transition induces aberrant glycosylation through hexosamine biosynthetic pathway activation. J Biol Chem 291(25):12917-12929. https://doi.org/ 10.1074/jbc.M116.729236

Luo C et al (2016) A PGC1 $\alpha$-mediated transcriptional axis suppresses melanoma metastasis. Nature 537(7620):422-426. https://doi. org/10.1038/nature19347

Marani M et al (2016) A pyrazolopyran derivative preferentially inhibits the activity of human cytosolic serine hydroxymethyltransferase and induces cell death in lung cancer cells. Oncotarget 7(4):4570-4583. https://doi.org/10.18632/oncotarget.6726

Marsico G et al (2018) Glycosylation and integrin regulation in cancer. Trends Cancer 4(8):537-552. https://doi.org/10.1016/j.trecan. 2018.05.009

Mishra RR, Kneitz S, Schartl M (2014) Comparative analysis of melanoma deregulated miRNAs in the medaka and Xiphophorus pigment cell cancer models. Comp Biochem Physiol C Toxicol 
Pharmacol 163:64-76. https://doi.org/10.1016/j.cbpc.2014.01. 002

Missiroli S et al (2020) Cancer metabolism and mitochondria: finding novel mechanisms to fight tumours. EBioMedicine 59:102943. https://doi.org/10.1016/j.ebiom.2020.102943

Moreno E et al (2018) The endocannabinoid system as a target in cancer diseases: are we there yet? Front Pharmacol 10:339. https:// doi.org/10.3389/fphar.2019.00339

Munkley J, Elliott DJ (2016) Hallmarks of glycosylation in cancer. Oncotarget 7(23):35478-35489. https://doi.org/10.18632/oncot arget. 8155

Nakamura M, Tokura Y (2011) Epithelial-mesenchymal transition in the skin. J Dermatol Sci 61(1):7-13. https://doi.org/10.1016/j. jdermsci.2010.11.015

Nardy AFFR et al (2016) The sweet side of immune evasion: role of glycans in the mechanisms of cancer progression. Front Oncol. https://doi.org/10.3389/fonc.2016.00054

Nath K et al (2018) Effect of differences in metabolic activity of melanoma models on response to lonidamine plus doxorubicin. Sci Rep 8(1):14654. https://doi.org/10.1038/s41598-018-33019-4

Nava-Molina L et al (2020) Novel CB1 receptor antagonist BAR-1 modifies pancreatic islet function and clinical parameters in prediabetic and diabetic mice. Nutr Diabetes 10(1):7. https://doi.org/ 10.1038/s41387-020-0110-0

Nesci S (2018) A lethal channel between the ATP synthase monomers. Trends Biochem Sci 43(5):311-313. https://doi.org/10.1016/j. tibs.2018.02.013

Ortonne J-P, Ballotti R (2000) Melanocyte biology and melanogenesis: what's new? J Dermatol Treat 11(1):15-26. https://doi.org/10. 1080/09546630050517621

Ozcan A, Ogun M (2015) Biochemistry of reactive oxygen and nitrogen species. In: Gowder SJT (ed) Basic principles and clinical significance of oxidative stress. InTech. https://doi.org/10.5772/ 61193

Paiardini A et al (2016) Differential 3-bromopyruvate inhibition of cytosolic and mitochondrial human serine hydroxymethyltransferase isoforms, key enzymes in cancer metabolic reprogramming. Biochim Biophys Acta Acta Proteins Proteom 1864(11):1506-1517. https://doi.org/10.1016/j.bbapap.2016. 08.010

Paone A et al (2014) SHMT1 knockdown induces apoptosis in lung cancer cells by causing uracil misincorporation. Cell Death Dis 5:e1525-e1525. https://doi.org/10.1038/cddis.2014.482

Parmenter TJ et al (2014) Response of BRAF-mutant melanoma to BRAF inhibition is mediated by a network of transcriptional regulators of glycolysis. Cancer Discov 4(4):423-433. https:// doi.org/10.1158/2159-8290.CD-13-0440

Pei Z et al (2012) Real-time analysis of the carbohydrates on cell surfaces using a QCM biosensor: a lectin-based approach. Biosens Bioelectron 35(1):200-205. https://doi.org/10.1016/j.bios.2012. 02.047

Peiris D et al (2012) A novel approach to determining the affinity of protein-carbohydrate interactions employing adherent cancer cells grown on a biosensor surface. Biosens Bioelectron 35(1):160-166. https://doi.org/10.1016/j.bios.2012.02.037

Peumans WJ, Van Damme E (1995) Lectins as plant defense proteins. Plant Physiol 109(2):347-352. https://doi.org/10.1104/pp.109.2. 347

Pijuan J et al (2019) In vitro cell migration, invasion, and adhesion assays: from cell imaging to data analysis. Front Cell Dev Biol 7:107. https://doi.org/10.3389/fcell.2019.00107

Pilawa B, Zdybel M, Chodurek E (2017) Application of electron paramagnetic resonance spectroscopy to examine free radicals in melanin polymers and the human melanoma malignum cells. In: Blumenberg M (ed) Melanin. InTech. https://doi.org/10.5772/ 67377
Pinho SS, Reis CA (2015) Glycosylation in cancer: mechanisms and clinical implications. Nat Rev Cancer 15(9):540-555. https://doi. org/10.1038/nrc3982

Pogoda K et al (2012) Depth-sensing analysis of cytoskeleton organization based on AFM data. Eur Biophys J 41(1):79-87. https://doi. org/10.1007/s00249-011-0761-9

Pokrywka M, Lityńska A (2012) Celując w czerniaka. Postępy Biologii Komórki. https://ruj.uj.edu.pl/xmlui/handle/item/25450. Accessed 18 Mar 2020

Potapenko IO et al (2010) Glycan gene expression signatures in normal and malignant breast tissue; possible role in diagnosis and progression. Mol Oncol 4(2):98-118. https://doi.org/10.1016/j. molonc.2009.12.001

Powell LD (2001) Inhibition of N-Linked glycosylation. Curr Protoc Immunol. https://doi.org/10.1002/0471142735.im0814s09

Przybyło M et al (2002) No title found. Cancer Cell Int 2(1):6. https:// doi.org/10.1186/1475-2867-2-6

Przybyło $M$ et al (2007) Identification of proteins bearing $\beta 1-6$ branched $N$-glycans in human melanoma cell lines from different progression stages by tandem mass spectrometry analysis. Biochim Biophys Acta Acta (BBA) Gen Subj 1770(9):1427-1435. https://doi.org/10.1016/j.bbagen.2007.05.006

Pucciarelli D et al (2016) Hypoxia increases the heterogeneity of melanoma cell populations and affects the response to vemurafenib. Mol Med Rep 13(4):3281-3288. https://doi.org/10.3892/mmr. 2016.4888

Ramer R, Schwarz R, Hinz B (2019) Modulation of the endocannabinoid system as a potential anticancer strategy. Front Pharmacol 10:430. https://doi.org/10.3389/fphar.2019.00430

Rankin EB, Nam J-M, Giaccia AJ (2016) Hypoxia: signaling the metastatic cascade. Trends Cancer 2(6):295-304. https://doi.org/10. 1016/j.trecan.2016.05.006

Rasola A, Bernardi P (2014) The mitochondrial permeability transition pore and its adaptive responses in tumor cells. Cell Calcium 56(6):437-445. https://doi.org/10.1016/j.ceca.2014.10.003

Rastrelli M et al (2014) Melanoma: epidemiology, risk factors, pathogenesis, diagnosis and classification. In Vivo (Athens, Greece) 28(6): 1005-1011

Ratnikov BI et al (2017) Metabolic rewiring in melanoma. Oncogene 36(2):147-157. https://doi.org/10.1038/onc.2016.198

Rodríguez-Enríquez S, Kaambre T, Moreno-Sánchez R (2020) Editorial: metabolic plasticity of cancer. Front Oncol 10:599723. https://doi.org/10.3389/fonc.2020.599723

Romero-Garcia S et al (2016) Lactate contribution to the tumor microenvironment: mechanisms, effects on immune cells and therapeutic relevance. Front Immunol. https://doi.org/10.3389/fimmu. 2016.00052

Ruocco MR et al (2019) Metabolic flexibility in melanoma: a potential therapeutic target. Semin Cancer Biol 59:187-207. https://doi. org/10.1016/j.semcancer.2019.07.016

Sarna M et al (2014) Cell elasticity is an important indicator of the metastatic phenotype of melanoma cells. Exp Dermatol 23(11):813818. https://doi.org/10.1111/exd.12535

Sarna M et al (2018) Nanomechanical phenotype of melanoma cells depends solely on the amount of endogenous pigment in the cells. Int J Mol Sci 19(2):607. https://doi.org/10.3390/ijms 19020607

Sarna M et al (2019) Melanin presence inhibits melanoma cell spread in mice in a unique mechanical fashion. Sci Rep 9(1):9280. https://doi.org/10.1038/s41598-019-45643-9

Schnaar RL, Kinoshita T (2017) Glycosphingolipids. In: Varki A et al. (eds) Essentials of glycobiology, 3rd edn. Cold Spring Harbor Laboratory Press, Cold Spring Harbor. http://www.ncbi.nlm.nih. gov/books/NBK453016/. Accessed 15 June 2020

Scolyer RA, Long GV, Thompson JF (2011) Evolving concepts in melanoma classification and their relevance to multidisciplinary 
melanoma patient care. Mol Oncol 5(2):124-136. https://doi.org/ 10.1016/j.molonc.2011.03.002

Scott DA et al (2011) Comparative metabolic flux profiling of melanoma cell lines: BEYOND THE WARBURG EFFECT. J Biol Chem 286(49):42626-42634. https://doi.org/10.1074/jbc.M111. 282046

Shankar Babu M et al (2018) Lapachol inhibits glycolysis in cancer cells by targeting pyruvate kinase M2. PLoS One 13(2):e0191419. https://doi.org/10.1371/journal.pone.0191419

Sharma LK, Lu J, Bai Y (2009) Mitochondrial respiratory complex I: structure, function and implication in human diseases. Curr Med Chem 16(10):1266-1277. https://doi.org/10.2174/09298 6709787846578

Silva-filho AF et al (2017) Glycobiology modifications in intratumoral hypoxia: the breathless side of glycans interaction. Cell Physiol Biochem 41(5):1801-1829. https://doi.org/10.1159/000471912

Slamenova D et al (2013) Comparison of biological processes induced in HepG2 cells by tert-butyl hydroperoxide (t-BHP) and hydroperoxide $\left(\mathrm{H}_{2} \mathrm{O}_{2}\right)$ : the influence of carvacrol. Mutat Res Genet Toxicol Environ Mutagen 757(1):15-22. https://doi.org/10.1016/j. mrgentox.2013.03.014

Slominski A, Zmijewski MA, Pawelek J (2012) L-tyrosine and L-dihydroxyphenylalanine as hormone-like regulators of melanocyte functions: L-tyrosine and L-DOPA as bioregulators. Pigment Cell Melanoma Res 25(1):14-27. https://doi.org/10.1111/j.1755148X.2011.00898.x

Slominski A et al (2014) The role of melanogenesis in regulation of melanoma behavior: melanogenesis leads to stimulation of HIF-1 $\alpha$ expression and HIF-dependent attendant pathways. Arch Biochem Biophys 563:79-93. https://doi.org/10.1016/j.abb.2014. 06.030

Śniegocka M et al (2018) Transplantable melanomas in hamsters and gerbils as models for human melanoma. Sensitization in melanoma radiotherapy-from animal models to clinical trials. Int $\mathbf{J}$ Mol Sci 19(4):1048. https://doi.org/10.3390/ijms19041048

Sobiepanek A (2017) Rola glikokoniugatów powierzchniowych w diagnostyce nowotworów złośliwych skóry. In: Zagadnienia aktualnie poruszane przez młodych naukowców. CREATIVETIME, pp 24-28

Sobiepanek A, Kobiela T (2018) Application of biosensors in cancer research. Rev Res Cancer Treat 4(1):4-12

Sobiepanek A, Kobiela T (2021) The study of the skin cells viscoelastic changes using QCM-D measurements. In: Ossandon M, Baker $\mathrm{H}$, Rasooly A (eds) Bioengineering technologies. Methods in molecular biology. Humana Press

Sobiepanek A et al (2017) AFM and QCM-D as tools for the distinction of melanoma cells with a different metastatic potential. Biosens Bioelectron 93:274-281. https://doi.org/10.1016/j.bios.2016.08. 088

Sobiepanek A et al (2020a) Different types of surface modification used for improving the adhesion and interactions of skin cells. Open Access J Biomed Sci 2(1):275-278. https://doi.org/10.38125/ OAJBS.000161

Sobiepanek A et al (2020b) A short guide on the selection of melanocytes and melanoma cells' isolation procedures for cancer research. Rev Res Cancer Treat 6(1):67-78

Sobiepanek A et al (2020c) Implementation of geneticin in the in vitro cell culture and in vivo studies. Rev Res Cancer Treat 6(1):79-87

Stanley P, Taniguchi N, Aebi M (2017) $N$-Glycans. In: Varki A et al (eds) Essentials of glycobiology, 3rd edn. Cold Spring Harbor Laboratory Press, Cold Spring Harbor. http://www.ncbi.nlm.nih. gov/books/NBK453020/. Accessed 16 June 2020

Stowell SR, Ju T, Cummings RD (2015) Protein glycosylation in cancer. Annu Rev Pathol 10(1):473-510. https://doi.org/10.1146/ annurev-pathol-012414-040438
Stubbs M, Griffiths JR (2010) The altered metabolism of tumors: HIF-1 and its role in the Warburg effect. Adv Enzyme Regul 50(1):4455. https://doi.org/10.1016/j.advenzreg.2009.10.027

Sweeney JG et al (2018) Loss of GCNT2/I-branched glycans enhances melanoma growth and survival. Nat Commun 9(1):3368. https:// doi.org/10.1038/s41467-018-05795-0

Taniguchi N, Kizuka Y (2015) Glycans and cancer. Adv Cancer Res. https://doi.org/10.1016/bs.acr.2014.11.001

Van Den Bossche K, Naeyaert J-M, Lambert J (2006) The quest for the mechanism of melanin transfer: revelations of the pigmentation synapse. Traffic 7(7):769-778. https://doi.org/10.1111/j.16000854.2006.00425.x

Vasconcelos-dos-Santos A et al (2015) Biosynthetic machinery involved in aberrant glycosylation: promising targets for developing of drugs against cancer. Front Oncol. https://doi.org/10. 3389/fonc. 2015.00138

Vazquez F et al (2013) PGC1 $\alpha$ expression defines a subset of human melanoma tumors with increased mitochondrial capacity and resistance to oxidative stress. Cancer Cell 23(3):287-301. https:// doi.org/10.1016/j.ccr.2012.11.020

Wang X et al (2014) 1H NMR metabolomics study of metastatic melanoma in C57BL/6J mouse spleen. Metabolomics 10(6):1129 1144. https://doi.org/10.1007/s11306-014-0652-z

Weder G et al (2014) 'Increased plasticity of the stiffness of melanoma cells correlates with their acquisition of metastatic properties. Nanomed Nanotechnol Biol Med 10(1):141-148. https://doi.org/ 10.1016/j.nano.2013.07.007

Wigerup C, Påhlman S, Bexell D (2016) Therapeutic targeting of hypoxia and hypoxia-inducible factors in cancer. Pharmacol Ther 164:152-169. https://doi.org/10.1016/j.pharmthera.2016.04.009

Wojtowicz $\mathrm{K}$ et al (2012) Inhibitors of $\mathrm{N}$-glycosylation as a potential tool for analysis of the mechanism of action and cellular localisation of glycoprotein P. Acta Biochim Pol 59(4):445-450

$\mathrm{Wu} \mathrm{AM}$ et al (2008) Lectins as tools in glycoconjugate research. Glycoconj J 26(8):899-913. https://doi.org/10.1007/ s10719-008-9119-7

Yakovleva ME, Safina GR, Danielsson B (2009) A study of glycoprotein-lectin interactions using quartz crystal microbalance. Anal Chim Acta 668(1):80-85. https://doi.org/10.1016/j.aca.2009.12. 004

Yallop CA, Svendsen I (2001) No title found. Cytotechnology 35(2):101-114. https://doi.org/10.1023/A:1017550902771

Yallop CA et al (2003) Characterisation of G418-induced metabolic load in recombinant $\mathrm{CHO}$ and BHK cells: effect on the activity and expression of central metabolic enzymes. Cytotechnology 42(2):87-99. https://doi.org/10.1023/B:CYTO.0000009821. $82741.8 \mathrm{c}$

Ząbczyńska M, Pocheć E (2015) The role of protein glycosylation in immune system. Postepy Biochem 61(2):129-137

Zaccagnino P et al (2011) Anandamide inhibits oxidative phosphorylation in isolated liver mitochondria. FEBS Lett 585(2):429-434. https://doi.org/10.1016/j.febslet.2010.12.032

Żądło AC (2019) Application of transition metal ions in a study of photoinduced modifications of melanin. Acta Biochim Pol. https:// doi.org/10.18388/abp.2018_2802

Zhang J, Zhang Q (2019) Using seahorse machine to measure OCR and ECAR in Cancer Cells. In: Haznadar M (ed) Cancer metabolism (methods in molecular biology). Springer, New York, pp 353-363. https://doi.org/10.1007/978-1-4939-9027-6_18

Zhou S et al (2017) LC-MS/MS analysis of permethylated $N$-glycans facilitating isomeric characterization. Anal Bioanal Chem 409(2):453-466. https://doi.org/10.1007/s00216-016-9996-8

Zhu N et al (2004) Melanoma cell migration is upregulated by tumour necrosis factor- $\alpha$ and suppressed by $\alpha$-melanocyte-stimulating hormone. Br J Cancer 90(7):1457-1463. https://doi.org/10.1038/ sj.bjc. 6601698 
Publisher's Note Springer Nature remains neutral with regard to jurisdictional claims in published maps and institutional affiliations. 